\title{
Common Fixed Point Theorems for Contractive Mappings of Integral Type in G-Metric Spaces and Applications
}

\author{
Fengrong Zhang $\mathbb{D},{ }^{1}$ Xiangshuai Zhang $\mathbb{D}^{2},{ }^{2}$ and Yan Hao $\mathbb{D}^{3}$ \\ ${ }^{1}$ School of Information Science and Engineering, Dalian Polytechnic University, Dalian, Liaoning 116034, China \\ ${ }^{2}$ Department of Mathematics, Liaoning Normal University, Dalian, Liaoning 116029, China \\ ${ }^{3}$ School of Mathematics and System Science, Shenyang Normal University, Shenyang, Liaoning 110034, China
}

Correspondence should be addressed to Yan Hao; haoyan8012@163.com

Received 14 November 2020; Revised 30 December 2020; Accepted 4 January 2021; Published 31 January 2021

Academic Editor: Huseyin Isik

Copyright () 2021 Fengrong Zhang et al. This is an open access article distributed under the Creative Commons Attribution License, which permits unrestricted use, distribution, and reproduction in any medium, provided the original work is properly cited.

Two common fixed point theorems for weakly compatible mappings satisfying contractive conditions of integral type in $G$-metric spaces are demonstrated. The results obtained in this paper generalize and differ from a few results in the literature and are used to prove the existence and uniqueness of common bounded and continuous solutions for certain functional equations and nonlinear Volterra integral equations. A nontrivial example is included.

\section{Introduction}

The Banach fixed point theorem which was first presented by Banach in 1922 is a significant result in fixed point theory. Because of its importance in proving the existence of solutions for functional equations, nonlinear Volterra integral equations and nonlinear integro-differential equations, this result has been extended in many different directions (see, e.g., [1-22] and the references cited therein). In particular, Rhoades [12] and Branciari [4] generalized the Banach fixed point theorem and gave the following fixed point theorems, respectively.

Theorem 1 (see [12]). Let $f$ be a mapping from a complete metric space $(X, d)$ into itself satisfying

$$
d(f x, f y) \leq d(x, y)-\varphi(d(x, y)), \quad \forall x, y \in X,
$$

where $\varphi \in \Phi_{4}$. Then, $f$ has a unique fixed point in $X$.

Theorem 2 (see [4]). Let $(X, d)$ be a complete metric space and $f: X \rightarrow X$ be a mapping satisfying

$$
\int_{0}^{d(f x, f y)} \varphi(t) d t \leq c \int_{0}^{d(x, y)} \varphi(t) d t, \quad \forall x, y \in X,
$$

where $\varphi \in \Phi_{1}$ and $c \in[0,1)$ is a constant. Then, $f$ has a unique fixed point $a \in X$ such that $\lim _{n \rightarrow \infty} f^{n} x=a$ for each $x \in X$.

In 2013, Gupta and Mani [21] obtained the existence and uniqueness of a fixed point for contractive mappings of an integral type in complete metric spaces by using iterative approximations. In 2007, Kumar et al. [6] proved a common fixed point theorem for a pair of compatible mappings satisfying a contractive inequality of integral type, which improves Theorem 2 .

Theorem 3 (see [6]). Let $(X, d)$ be a complete metric space and $f, g: X \rightarrow X$ be compatible mappings such that

$$
\begin{gathered}
f(X) \subseteq g(X), g \text { is continuous, } \\
\int_{0}^{d(f x, f y)} \varphi(t) d t \leq c \int_{0}^{d(g x, g y)} \varphi(t) d t, \quad \forall x, y \in X,
\end{gathered}
$$

where $\varphi \in \Phi_{1}$ and $c \in[0,1)$ is a constant. Then, $f$ and $g$ have a unique common fixed point in $X$.

In 2006, Mustafa and Sims [9] introduced a new concept of generalized metric space called $G$-metric space. From then 
on, lots of research works have been carried out on generalizing contractive conditions for different contractive mappings satisfying various known properties in $G$-metric spaces [1-3, $5,10,11,13,15,19,20]$. In 2018, Gupta et al. [19] proved some fixed point theorems for the functions satisfying $\phi$ -contraction and mixed $g$-monotone property in $G$-metric spaces. In 2015, Gupta and Deep [20] gave a few common fixed point theorems using the property E.A. in the setting of $G$-metric and fuzzy metric spaces by taking a set of three conditions for self-mappings. In 2011, Aydi [1] proved a fixed point theorem for mappings satisfying a $(\psi, \phi)$-weakly contractive condition in $G$-metric spaces.

Theorem 4 (see [1]). Let $(X, G)$ be a complete G-metric space and $f$ be a mapping from $X$ into itself satisfying

$$
\psi(G(f x, f y, f z)) \leq \psi(G(x, y, z))-\phi(G(x, y, z)), \quad \forall x, y, z \in X,
$$

where $\psi, \phi \in \Phi_{2}$. Then, $f$ has a unique fixed point $u \in X$ and $f$ is $G$-continuous at $u$.

In 2012, Aydi [2] obtained the following common fixed point theorem for a pair of mappings involving a contractive condition of integral type in $G$-metric spaces.

Theorem 5 (see [2]). Let $(X, G)$ be a G-metric space and $f, g$ be two mappings from $X$ into itself such that

$$
\int_{0}^{G(f x, f y, f z)} \varphi(t) d t \leq \alpha \int_{0}^{G(g x, g y, g z)} \varphi(t) d t, \quad \forall x, y, z \in X,
$$

where $\varphi \in \Phi_{1}$ and $\alpha \in[0,1)$ is a constant. If $f(X) \subseteq g(X)$ and $g(X)$ is a complete subset of $X$, then $f$ and $g$ have a unique point of coincidence in X. Moreover, if $f$ and $g$ are weakly compatible, then $f$ and $g$ have a unique common fixed point.

The objective of this paper is both to introduce two new classes of contractive mappings of integral type in the setting of $G$-metric spaces and to prove the existence and uniqueness of points of coincidence and common fixed points for these mappings. Our results extend Theorem 5, are different from Theorem 4, and are used to show solvability of the functional equations arising in dynamic programming and nonlinear Volterra integral equations. A nontrivial example is given.

\section{Preliminaries}

Throughout this paper, $\mathbb{N}$ denotes the set of all positive integers, $\mathbb{N}_{0}=\{0\} \cup \mathbb{N}, \mathbb{R}^{+}=[0,+\infty)$, and $\mathbb{R}=(-\infty,+\infty)$. Put

$$
\Phi_{1}=\left\{\varphi \mid \varphi: \mathbb{R}^{+} \rightarrow \mathbb{R}^{+}\right.
$$

is Lebesgue integrable and summable on each compact subset of $\mathbb{R}^{+}$and

$$
\left.\int_{0}^{\epsilon} \varphi(t) d t>0, \forall \epsilon>0\right\}
$$

$\Phi_{2}=\left\{\varphi \mid \varphi: \mathbb{R}^{+} \rightarrow \mathbb{R}^{+}\right.$

is a continuous and nondecreasing function such that $\varphi(t)=0$

if and only if $t=0\}$,

$\Phi_{3}=\left\{\varphi \mid \varphi: \mathbb{R}^{+} \rightarrow \mathbb{R}^{+}\right.$satisfies that

$\liminf _{n \rightarrow \infty} \varphi\left(a_{n}\right)>0 \leftrightarrow \liminf _{n \rightarrow \infty} a_{n}>0$

for each $\left.\left\{a_{n}\right\}_{n \in \mathbb{N}} \subset \mathbb{R}^{+}\right\}$,

$\Phi_{4}=\left\{\varphi \mid \varphi \in \Phi_{2}\right.$ and $\left.\lim _{t \rightarrow+\infty} \varphi(t)=+\infty\right\}$

$\Phi_{5}=\left\{\varphi \mid \varphi \in \Phi_{2}\right.$ and $\left.\varphi\left(t_{1}+t_{2}\right) \leq \varphi\left(t_{1}\right)+\varphi\left(t_{2}\right), \quad \forall t_{1}, t_{2} \in \mathbb{R}^{+}\right\}$

Definition 6 (see [9]). Let $X$ be a nonempty set and $G: X \times$ $X \times X \rightarrow \mathbb{R}^{+}$be a function satisfying the following properties:

(G1) $G(x, y, z)=0$ if $x=y=z$,

(G2) $0<G(x, x, y)$ for all $x, y \in X$ with $x \neq y$,

(G3) $G(x, x, y) \leq G(x, y, z)$ for all $x, y, z \in X$ with $y \neq z$,

(G4) $G(x, y, z)=G(x, z, y)=G(y, z, x)=\cdots$, symmetry in all three variables,

(G5) $G(x, y, z) \leq G(x, a, a)+G(a, y, z)$ for all $x, y, z, a \in X$.

The function $G$ is called a $G$-metric in $X$, and the pair $(X, G)$ is called a $G$-metric space.

Definition 7 (see [9]). Let $(X, G)$ be a $G$-metric space and $\left\{x_{n}\right\}_{n \in \mathbb{N}}$ be a sequence of points of $X$. The sequence $\left\{x_{n}\right\}_{n \in \mathbb{N}}$ is $G$-convergent to $x \in X$ if

$$
\lim _{n, m \rightarrow \infty} G\left(x, x_{n}, x_{m}\right)=0,
$$

that is, for any $\varepsilon>0$, there exists $N \in \mathbb{N}$ such that

$$
G\left(x, x_{n}, x_{m}\right)<\varepsilon, \quad \forall m, n \geq N .
$$

The point $x$ is called the limit of the sequence $\left\{x_{n}\right\}_{n \in \mathbb{N}}$ and write $x_{n} \rightarrow x$ or $\lim _{n \rightarrow \infty} x_{n}=x$.

Lemma 8 (see [9]). Let $(X, G)$ be a G-metric space. Then, the following statements are equivalent:

(1) $\left\{x_{n}\right\}_{n \in \mathbb{N}}$ is G-convergent to $x$,

(2) $G\left(x_{n}, x_{n}, x\right) \rightarrow 0$ as $n \rightarrow \infty$,

(3) $G\left(x_{n}, x, x\right) \rightarrow 0$ as $n \rightarrow \infty$,

(4) $G\left(x_{n}, x_{m}, x\right) \rightarrow 0$ as $n, m \rightarrow \infty$.

Definition 9 (see [9]). Let $(X, G)$ be a $G$-metric space. A sequence $\left\{x_{n}\right\}_{n \in \mathbb{N}}$ in $X$ is called $G$-Cauchy if for every $\varepsilon>0$, there is $N \in \mathbb{N}$ such that 


$$
G\left(x_{l}, x_{n}, x_{m}\right)<\varepsilon, \quad \forall l, n, m \geq N,
$$

that is, $G\left(x_{l}, x_{n}, x_{m}\right) \rightarrow 0$ asl, $n, m \rightarrow \infty$.

Lemma 10 (see [9]). Let $(X, G)$ be a G-metric space. Then, the following statements are equivalent:

(1) $\left\{x_{n}\right\}_{n \in \mathbb{N}}$ is G-Cauchy,

(2) For every $\varepsilon>0$, there exists $N \in \mathbb{N}$ such that $G\left(x_{n}, x_{n}\right.$, $\left.x_{m}\right)<\varepsilon$ for all $n, m \geq N$.

Definition 11 (see [9]). A $G$-metric space $(X, G)$ is called $G$ -complete if every $G$-Cauchy sequence is $G$-convergent in $(X, G)$.

Lemma 12 (see [9]). Let $(X, G)$ be a G-metric space. Then, the function $G(x, y, z)$ is jointly continuous in all three of its variables.

Lemma 13 (see [9]). Let $(X, G)$ be a G-metric space. Then,

$$
\begin{gathered}
G(x, y, y) \leq 2 G(y, x, x), \quad \forall x, y \in X, \\
|G(x, y, z)-G(x, y, a)| \leq \max \{G(a, z, z), G(z, a, a)\}, \quad \forall x, y, z, a \in X .
\end{gathered}
$$

Definition 14 (see [14]). LetS and $T$ be self-mappings of a nonempty set $X$.

(1) A point $x \in X$ is said to be a fixed point of $T$ if $T x=x$.

(2) A point $x \in X$ is said to be a coincidence point of $S$ and $T$ if $T x=S x$ and $w=S x=T x$ is said to be a point of coincidence of $S$ and $T$.

(3) A point $x \in X$ is said to be a common fixed point of $S$ and $T$ if $x=T x=S x$.

Definition 15. A pair of self-mappings $f$ and $g$ in a $G$-metric space $(X, G)$ are said to be weakly compatible if for any $x \in X$, the equality $f x=g x$ gives that $f g x=g f x$.

Lemma 16 (see [14]). Let $X$ be a nonempty set and $f, g: X$ $\rightarrow X$ be weakly compatible mappings. If $f$ and $g$ have a unique point of coincidence $w \in X$, then $w$ is the unique common fixed point of $f$ and $g$.

Lemma 17 (see [7]). Let $\varphi \in \Phi_{1}$ and $\left\{r_{n}\right\}_{n \in \mathbb{N}}$ be a nonnegative sequence with $\lim _{n \rightarrow \infty} r_{n}=a$. Then,

$$
\lim _{n \rightarrow \infty} \int_{0}^{r_{n}} \varphi(t) d t=\int_{0}^{a} \varphi(t) d t .
$$

Lemma 18 (see [8]). Let $\varphi \in \Phi_{3}$. Then, $\varphi(t)>0$ if and only if $t>0$.

\section{Main Results}

Now, we study the existence and uniqueness of points of coincidence and common fixed points for contractive mappings (12) and (51) below in $G$-metric spaces, respectively.

Theorem 19. Let $(X, G)$ be a $G$-metric space, $f$ and $g: X \rightarrow X$ be two mappings satisfying

$$
\begin{gathered}
\psi\left(\int_{0}^{G(f x, f y, f z)} \varphi(t) d t\right) \leq \psi\left(\int_{0}^{M_{1}(x, y, z)} \varphi(t) d t\right) \\
-\phi\left(\int_{0}^{M_{1}(x, y, z)} \varphi(t) d t\right), \quad \forall x, y, z \in X,
\end{gathered}
$$

where $(\varphi, \psi, \phi) \in \Phi_{1} \times \Phi_{2} \times \Phi_{3}$ and

$$
\begin{aligned}
M_{1}(x, y, z)= & \max \left\{G(g x, g y, g z), \frac{[1+G(g x, g x, f x)] G(g y, g y, f z)}{2+G(g x, f x, f y)},\right. \\
& \frac{[1+G(g x, g x, f x)] G(g z, g z, f y)}{2+G(g x, f x, f z)}, \\
& \frac{[1+G(g y, g y, f y)] G(g z, g z, f x)}{2+G(g y, f y, f z)}, \\
& \frac{[1+G(g y, g y, f y)] G(g x, g x, f z)}{2[1+G(g y, f x, f y)]} \\
& \frac{[1+G(g z, g z, f z)] G(g y, g y, f x)}{2[1+G(g z, f y, f z)]} \\
& \frac{[1+G(g z, g z, f z)] G(g x, g x, f y)}{2[1+G(g z, f x, f z)]} \\
& \frac{[1+G(g x, g y, g z)] G(f x, f y, f z)}{1+G(g z, f y, f y)+G(g x, g y, g z)} \\
& \frac{[1+G(g x, g y, g z)] G(f x, f y, f z)}{1+G(g y, f x, f x)+G(g x, g y, g z)} \\
& \left.\frac{[1+G(g x, g y, g z)] G(f x, f y, f z)}{1+G(g x, f z, f z)+G(g x, g y, g z)}\right\}
\end{aligned}
$$

If $f(X) \subseteq g(X)$ and $g(X)$ is a complete subset of $X$, then $f$ and $g$ have a unique point of coincidence in $X$. Furthermore, if $f$ and $g$ are weakly compatible mappings, then $f$ and $g$ have a unique common fixed point in $X$.

Proof. Let $x_{0}$ be an arbitrary point in $X$. Since $f(X) \subseteq g(X)$, it follows that there exists a sequence $\left\{x_{n}\right\}_{n \in \mathbb{N}_{0}}$ in $X$ satisfying

$$
f x_{n}=g x_{n+1}, \quad \forall n \in \mathbb{N}_{0} .
$$

Put $G_{n}=G\left(f x_{n}, f x_{n+1}, f x_{n+2}\right)$ for all $n \in \mathbb{N}_{0}$. Assume that $f x_{n_{0}}=f x_{n_{0}+1}$ for some $n_{0} \in \mathbb{N}_{0}$. It is clear that $g x_{n_{0}+1}=f x_{n_{0}+1}$, that is, $f x_{n_{0}+1}$ is a point of coincidence of $f$ and $g$. Assume that $f x_{n} \neq f x_{n+1}$ for all $n \in \mathbb{N}_{0}$. Clearly, $G_{n}>0$ for all $n \in \mathbb{N}_{0}$. By 
virtue of (G3)-(G5) and (14), we observe that

$$
\begin{aligned}
& \frac{\left[1+G\left(g x_{n+1}, g x_{n+1}, f x_{n+1}\right)\right] G\left(g x_{n}, g x_{n}, f x_{n+2}\right)}{2\left[1+G\left(g x_{n+1}, f x_{n}, f x_{n+1}\right)\right]} \\
& =\frac{\left[1+G\left(f x_{n}, f x_{n}, f x_{n+1}\right)\right] G\left(f x_{n-1}, f x_{n-1}, f x_{n+2}\right)}{2\left[1+G\left(f x_{n}, f x_{n}, f x_{n+1}\right)\right]} \\
& =\frac{G\left(f x_{n-1}, f x_{n-1}, f x_{n+2}\right)}{2}=\frac{G\left(f x_{n+2}, f x_{n-1}, f x_{n-1}\right)}{2} \\
& \leq \frac{G\left(f x_{n+2}, f x_{n}, f x_{n}\right)+G\left(f x_{n}, f x_{n-1}, f x_{n-1}\right)}{2} \\
& =\frac{G\left(f x_{n}, f x_{n}, f x_{n+2}\right)+G\left(f x_{n-1}, f x_{n-1}, f x_{n}\right)}{2} \\
& \leq \frac{G\left(f x_{n}, f x_{n+2}, f x_{n+1}\right)+G\left(f x_{n-1}, f x_{n}, f x_{n+1}\right)}{2} \\
& =\frac{G_{n-1}+G_{n}}{2}, \quad \forall n \in \mathbb{N} .
\end{aligned}
$$

Set

$$
\begin{aligned}
m_{1}\left(x_{n}, x_{n+1}, x_{n+2}\right)= & \max \left\{\frac{\left[1+G\left(g x_{n}, g x_{n}, f x_{n}\right)\right] G\left(g x_{n+1}, g x_{n+1}, f x_{n+2}\right)}{2+G\left(g x_{n}, f x_{n}, f x_{n+1}\right)},\right. \\
& \frac{\left[1+G\left(g x_{n}, g x_{n}, f x_{n}\right)\right] G\left(g x_{n+2}, g x_{n+2}, f x_{n+1}\right)}{2+G\left(g x_{n}, f x_{n}, f x_{n+2}\right)}, \\
& \frac{\left[1+G\left(g x_{n+1}, g x_{n+1}, f x_{n+1}\right)\right] G\left(g x_{n+2}, g x_{n+2}, f x_{n}\right)}{2+G\left(g x_{n+1}, f x_{n+1}, f x_{n+2}\right)}, \\
& \frac{\left[1+G\left(g x_{n+1}, g x_{n+1}, f x_{n+1}\right)\right] G\left(g x_{n}, g x_{n}, f x_{n+2}\right)}{2\left[1+G\left(g x_{n+1}, f x_{n}, f x_{n+1}\right)\right]}, \\
& \frac{\left[1+G\left(g x_{n+2}, g x_{n+2}, f x_{n+2}\right)\right] G\left(g x_{n+1}, g x_{n+1}, f x_{n}\right)}{2\left[1+G\left(g x_{n+2}, f x_{n+1}, f x_{n+2}\right)\right]}, \\
& \left.\frac{\left[1+G\left(g x_{n+2}, g x_{n+2}, f x_{n+2}\right)\right] G\left(g x_{n}, g x_{n}, f x_{n+1}\right)}{2\left[1+G\left(g x_{n+2}, f x_{n}, f x_{n+2}\right)\right]}\right\}, \quad \forall n \in \mathbb{N},
\end{aligned}
$$

which together with (15) yields that

$$
\begin{aligned}
m_{1}\left(x_{n}, x_{n+1}, x_{n+2}\right)= & \max \left\{\frac{\left[1+G\left(f x_{n-1}, f x_{n-1}, f x_{n}\right)\right] G\left(f x_{n}, f x_{n}, f x_{n+2}\right)}{2+G\left(f x_{n-1}, f x_{n}, f x_{n+1}\right)},\right. \\
& \frac{\left[1+G\left(f x_{n-1}, f x_{n-1}, f x_{n}\right)\right] G\left(f x_{n+1}, f x_{n+1}, f x_{n+1}\right)}{2+G\left(f x_{n-1}, f x_{n}, f x_{n+2}\right)}, \\
& \frac{\left[1+G\left(f x_{n}, f x_{n}, f x_{n+1}\right)\right] G\left(f x_{n+1}, f x_{n+1}, f x_{n}\right)}{2+G\left(f x_{n}, f x_{n+1}, f x_{n+2}\right)}, \\
& \frac{\left[1+G\left(f x_{n}, f x_{n}, f x_{n+1}\right)\right] G\left(f x_{n-1}, f x_{n-1}, f x_{n+2}\right)}{2\left[1+G\left(f x_{n}, f x_{n}, f n+1\right)\right]}, \\
& \frac{\left[1+G\left(f x_{n+1}, f x_{n+1}, f x_{n+2}\right)\right] G\left(f x_{n}, f x_{n}, f x_{n}\right)}{2\left[1+G\left(f x_{n+1}, f x_{n+1}, f x_{n+2}\right)\right]}, \\
& \left.\frac{\left[1+G\left(f x_{n+1}, f x_{n+1}, f x_{n+2}\right)\right] G\left(f x_{n-1}, f x_{n-1}, f x_{n+1}\right)}{2\left[1+G\left(f x_{n+1}, f x_{n}, f x_{n+2}\right)\right]}\right\} \\
\leq & \max \left\{\frac{\left(1+G_{n-1}\right) G_{n}}{2+G_{n-1}}, 0, \frac{\left(1+G_{n}\right) G_{n-1}, G_{n-1}+G_{n}}{2+G_{n}}, 0,\right. \\
& \left.\frac{\left(1+2 G_{n}\right) G_{n-1}}{2\left(1+G_{n}\right)}\right\} \leq \max \left\{G_{n-1}, G_{n}\right\}, \quad \forall n \in \mathbb{N} .
\end{aligned}
$$

In light of (G1), (G3), (G5), and (13)-(17), we get that

$$
\begin{aligned}
M_{1}\left(x_{n}, x_{n+1}, x_{n+2}\right)= & \max \left\{G\left(g x_{n}, g x_{n+1}, g x_{n+2}\right), m_{1}\left(x_{n}, x_{n+1}, x_{n+2}\right),\right. \\
& \frac{\left[1+G\left(g x_{n}, g x_{n+1}, g x_{n+2}\right)\right] G\left(f x_{n}, f x_{n+1}, f x_{n+2}\right)}{1+G\left(g x_{n+2}, f x_{n+1}, f x_{n+1}\right)+G\left(g x_{n}, g x_{n+1}, g x_{n+2}\right)}, \\
& \frac{\left[1+G\left(g x_{n}, g x_{n+1}, g x_{n+2}\right)\right] G\left(f x_{n}, f x_{n+1}, f x_{n+2}\right)}{1+G\left(g x_{n+1}, f x_{n}, f x_{n}\right)+G\left(g x_{n}, g x_{n+1}, g x_{n+2}\right)}, \\
& \frac{\left[1+G\left(g x_{n}, g x_{n+1}, g x_{n+2}\right)\right] G\left(f x_{n}, f x_{n+1}, f x_{n+2}\right)}{\left.1+G\left(g x_{n}, f x_{n+2}, f x_{n+2}\right)+G\left(g x_{n}, g x_{n+1}, g x_{n+2}\right)\right\}}, \\
= & \max \left\{G\left(f x_{n-1}, f x_{n}, f x_{n+1}\right), m_{1}\left(x_{n}, x_{n+1}, x_{n+2}\right),\right. \\
& \frac{\left[1+G\left(f x_{n-1}, f x_{n}, f x_{n+1}\right)\right] G\left(f x_{n}, f x_{n+1}, f x_{n+2}\right)}{1+G\left(f x_{n+1}, f x_{n+1}, f x_{n+1}\right)+G\left(f x_{n-1}, f x_{n}, f x_{n+1}\right)}, \\
& \frac{\left[1+G\left(f x_{n-1}, f x_{n}, f x_{n+1}\right)\right] G\left(f x_{n}, f x_{n+1}, f x_{n+2}\right)}{1+G\left(f x_{n}, f x_{n}, f x_{n}\right)+G\left(f x_{n-1}, f x_{n}, f x_{n+1}\right)}, \\
& \left.\frac{\left[1+G\left(f x_{n-1}, f x_{n}, f x_{n+1}\right)\right] G\left(f x_{n}, f x_{n+1}, f x_{n+2}\right)}{1+G\left(f x_{n-1}, f x_{n+2}, f x_{n+2}\right)+G\left(f x_{n-1}, f x_{n}, f x_{n+1}\right)}\right\} \\
= & \max \left\{G_{n-1}, m_{1}\left(x_{n}, x_{n+1}, x_{n+2}\right), G_{n}, G_{n},\right. \\
& \left.\frac{\left(1+G_{n-1}\right) G_{n}}{1+G\left(f x_{n-1}, f x_{n+2}, f x_{n+2}\right)+G_{n-1}}\right\} \\
= & \max \left\{G_{n-1}, G_{n}\right\}, \quad \forall n \in \mathbb{N} .
\end{aligned}
$$

Now we assert that $G_{n} \leq G_{n-1}, \forall n \in \mathbb{N}$. Suppose that there exists some $n_{0} \in \mathbb{N}$ satisfying $G_{n_{0}}>G_{n_{0}-1}$. It follows from (12), $(\varphi, \psi, \phi) \in \Phi_{1} \times \Phi_{2} \times \Phi_{3}$ and Lemma 18 , we infer that

$$
\begin{aligned}
\psi\left(\int_{0}^{G_{n_{0}}} \varphi(t) d t\right)= & \psi\left(\int_{0}^{G\left(f x_{n_{0}}, f x_{n_{0}+1}, f x_{n_{0}+2}\right)} \varphi(t) d t\right) \\
\leq & \psi\left(\int_{0}^{M_{1}\left(x_{n_{0}}, x_{n_{0}+1}, x_{n_{0}+2}\right)} \varphi(t) d t\right) \\
& -\phi\left(\int_{0}^{M_{1}\left(x_{n_{0}}, x_{n_{0}+1}, x_{n_{0}+2}\right)} \varphi(t) d t\right) \\
= & \psi\left(\int_{0}^{G_{n_{0}}} \varphi(t) d t\right)-\phi\left(\int_{0}^{G_{n_{0}}} \varphi(t) d t\right) \\
< & \psi\left(\int_{0}^{G_{n_{0}}} \varphi(t) d t\right),
\end{aligned}
$$

which is a contradiction. Therefore, $G_{n} \leq G_{n-1}$ for all $n \in \mathbb{N}$ and

$$
M_{1}\left(x_{n}, x_{n+1}, x_{n+2}\right)=G_{n-1}, \quad \forall n \in \mathbb{N} .
$$

It is apparent that the sequence $\left\{G_{n}\right\}_{n \in \mathbb{N}_{0}}$ is nonincreasing and bounded, which implies that there exists $r$ with

$$
\lim _{n \rightarrow \infty} G_{n}=r \geq 0
$$

Now, we demonstrate that $r=0$. Suppose that $r>0$. On account of (12), (20), and (21), $(\varphi, \psi, \phi) \in \Phi_{1} \times \Phi_{2} \times \Phi_{3}$ and Lemma 17, we deduce that 


$$
\begin{aligned}
\psi\left(\int_{0}^{r} \varphi(t) d t\right)= & \limsup _{n \rightarrow \infty} \psi\left(\int_{0}^{G_{n}} \varphi(t) d t\right) \\
= & \limsup _{n \rightarrow \infty} \psi\left(\int_{0}^{G\left(f x_{n}, \mathrm{x}_{n+1}, x_{n+2}\right)} \varphi(t) d t\right) \\
\leq & \limsup _{n \rightarrow \infty}\left[\psi\left(\int_{0}^{M_{1}\left(x_{n}, x_{n+1}, x_{n+2}\right)} \varphi(t) d t\right)\right. \\
& \left.-\phi\left(\int_{0}^{M_{1}\left(x_{n}, x_{n+1}, x_{n+2}\right)} \varphi(t) d t\right)\right] \\
= & \limsup _{n \rightarrow \infty}\left[\psi\left(\int_{0}^{G_{n-1}} \varphi(t) d t\right)-\phi\left(\int_{0}^{G_{n-1}} \varphi(t) d t\right)\right] \\
\leq & \limsup _{n \rightarrow \infty} \psi\left(\int_{0}^{G_{n-1}} \varphi(t) d t\right)-\liminf _{n \rightarrow \infty} \phi\left(\int_{0}^{G_{n-1}} \varphi(t) d t\right) \\
= & \psi\left(\int_{0}^{r} \varphi(t) d t\right)-\liminf _{n \rightarrow \infty} \phi\left(\int_{0}^{G_{n-1}} \varphi(t) d t\right) \\
< & \psi\left(\int_{0}^{r} \varphi(t) d t\right),
\end{aligned}
$$

which is impossible. Thus, $r=0$. That is,

$$
\lim _{n \rightarrow \infty} G_{n}=0
$$

It follows from (G3), (G4), and (23) that

$$
0 \leq G\left(f x_{n-1}, f x_{n-1}, f x_{n}\right) \leq G\left(f x_{n-1}, f x_{n}, f x_{n+1}\right) \rightarrow 0 \text { as } n \rightarrow \infty,
$$

$0 \leq G\left(f x_{n-1}, f x_{n}, f x_{n}\right)=G\left(f x_{n}, f x_{n}, f x_{n-1}\right) \leq G\left(f x_{n}, f x_{n-1}, f x_{n-2}\right) \rightarrow 0$ as $n \rightarrow \infty$,

which yield that

$$
\lim _{n \rightarrow \infty} G\left(f x_{n-1}, f x_{n-1}, f x_{n}\right)=\lim _{n \rightarrow \infty} G\left(f x_{n-1}, f x_{n}, f x_{n}\right)=0 .
$$

Next, we verify that $\left\{f x_{n}\right\}_{n \in \mathbb{N}_{0}}$ is a G-Cauchy sequence. Suppose that $\left\{f x_{n}\right\}_{n \in \mathbb{N}_{0}}$ is not a G-Cauchy sequence. It follows from Lemma 10 that there exist a constant $\varepsilon>0$ and two subsequences $\left\{f x_{m(k)}\right\}_{k \in \mathbb{N}}$ and $\left\{f x_{n(k)}\right\}_{k \in \mathbb{N}}$ of $\left\{f x_{n}\right\}_{n \in \mathbb{N}_{0}}$ such that $n(k)$ is minimal in the sense that

$k<m(k)<n(k)<m(k+1)$ and $G\left(f x_{m(k)}, f x_{m(k)}, f x_{n(k)}\right)>\varepsilon, \quad \forall k \in \mathbb{N}$,

which means that $G\left(f x_{m(k)}, f x_{m(k)}, f x_{n(k)-1}\right) \leq \varepsilon$ for all $k \in \mathbb{N}$.
By means of (G3)-(G5) and Lemma 13, we deduce that

$$
\begin{aligned}
& \varepsilon<G\left(f x_{m(k)}, f x_{m(k)}, f x_{n(k)}\right)= G\left(f x_{n(k)}, f x_{m(k)}, f x_{m(k)}\right) \\
& \leq G\left(f x_{n(k)}, f x_{n(k)-1}, f x_{n(k)-1}\right) \\
&+G\left(f x_{n(k)-1}, f x_{m(k)}, f x_{m(k)}\right) \\
& \leq \varepsilon+G\left(f x_{n(k)}, f x_{n(k)-1}, f x_{n(k)-1}\right) \\
& \leq \varepsilon+G_{n(k)-1}, \quad \forall k \in \mathbb{N}, \\
&\left|G\left(f x_{n(k)}, f x_{m(k)-1}, f x_{m(k)}\right)-G\left(f x_{m(k)}, f x_{m(k)}, f x_{n(k)}\right)\right| \\
& \leq \max \left\{G\left(f x_{m(k)-1}, f x_{m(k)}, f x_{m(k)}\right), G\left(f x_{m(k)}, f x_{m(k)-1}, f x_{m(k)-1}\right)\right\} \\
& \leq 2 G\left(f x_{m(k)-1}, f x_{m(k)}, f x_{m(k)+1}\right)=2 G_{m(k)-1}, \quad \forall k \in \mathbb{N},
\end{aligned}
$$

$$
\begin{aligned}
& \left|G\left(f x_{m(k)-1}, f x_{m(k)-1}, f x_{n(k)}\right)-G\left(f x_{n(k)}, f x_{m(k)-1}, f x_{m(k)}\right)\right| \\
& \quad \leq \max \left\{G\left(f x_{m(k)-1}, f x_{m(k)}, f x_{m(k)}\right), G\left(f x_{m(k)}, f x_{m(k)-1}, f x_{m(k)-1}\right)\right\} \\
& \quad \leq 2 G\left(f x_{m(k)-1}, f x_{m(k)}, f x_{m(k)+1}\right)=2 G_{m(k)-1}, \quad \forall k \in \mathbb{N},
\end{aligned}
$$

$$
\begin{aligned}
& \left|G\left(f x_{m(k)-1}, f x_{m(k)-1}, f x_{n(k)-1}\right)-G\left(f x_{m(k)-1}, f x_{m(k)-1}, f x_{n(k)}\right)\right| \\
& \quad \leq \max \left\{G\left(f x_{n(k)-1}, f x_{n(k)}, f x_{n(k)}\right), G\left(f x_{n(k)}, f x_{n(k)-1}, f x_{n(k)-1}\right)\right\} \\
& \quad \leq 2 G\left(f x_{n(k)-1}, f x_{n(k)}, f x_{n(k)+1}\right)=2 G_{n(k)-1}, \quad \forall k \in \mathbb{N},
\end{aligned}
$$

$$
\begin{aligned}
& \left|G\left(f x_{m(k)}, f x_{m(k)}, f x_{n(k)-1}\right)-G\left(f x_{m(k)}, f x_{m(k)}, f x_{n(k)}\right)\right| \\
& \quad \leq \max \left\{G\left(f x_{n(k)}, f x_{n(k)-1}, f x_{n(k)-1}\right), G\left(f x_{n(k)-1}, f x_{n(k)}, f x_{n(k)}\right)\right\} \\
& \quad \leq 2 G\left(f x_{n(k)-1}, f x_{n(k)}, f x_{n(k)+1}\right)=2 G_{n(k)-1}, \quad \forall k \in \mathbb{N}
\end{aligned}
$$

$$
\begin{aligned}
& \left|G\left(f x_{n(k)-1}, f x_{m(k)-1}, f x_{m(k)}\right)-G\left(f x_{m(k)}, f x_{m(k)}, f x_{n(k)-1}\right)\right| \\
& \quad \leq \max \left\{G\left(f x_{m(k)}, f x_{m(k)-1}, f x_{m(k)-1}\right), G\left(f x_{m(k)-1}, f x_{m(k)}, f x_{m(k)}\right)\right\} \\
& \quad \leq 2 G\left(f x_{m(k)-1}, f x_{m(k)}, f x_{m(k)+1}\right)=2 G_{m(k)-1}, \quad \forall k \in \mathbb{N} .
\end{aligned}
$$

Letting $k \rightarrow \infty$ in (27)-(32) and using (23) and (25), we obtain that

$$
\begin{aligned}
\varepsilon & =\lim _{k \rightarrow \infty} G\left(f \mathrm{x}_{m(k)}, f x_{m(k)}, f x_{n(k)}\right)=\lim _{k \rightarrow \infty} G\left(f x_{n(k)}, f x_{m(k)-1}, f x_{m(k)}\right) \\
& =\lim _{k \rightarrow \infty} G\left(f x_{m(k)-1}, f x_{m(k)-1}, f x_{n(k)}\right)=\lim _{k \rightarrow \infty} G\left(f x_{m(k)-1}, f x_{m(k)-1}, f x_{n(k)-1}\right) \\
& =\lim _{k \rightarrow \infty} G\left(f x_{m(k)}, f x_{m(k)}, f x_{n(k)-1}\right)=\lim _{k \rightarrow \infty} G\left(f x_{n(k)-1}, f x_{m(k)-1}, f x_{m(k)}\right) .
\end{aligned}
$$


In view of (G3)-(G5) and Lemma 13, we infer that

$$
\begin{aligned}
G\left(f x_{n(k)-1}, f x_{n(k)-1}, f x_{m(k)}\right)= & G\left(f x_{m(k)}, f x_{n(k)-1}, f x_{n(k)-1}\right) \\
\leq & G\left(f x_{m(k)}, f x_{n(k)}, f x_{n(k)}\right) \\
& +G\left(f x_{n(k)}, f x_{n(k)-1}, f x_{n(k)-1}\right) \\
\leq & G\left(f x_{m(k)}, f x_{m(k)-1}, f x_{n(k)}\right) \\
& +G\left(f x_{n(k)+1}, f x_{n(k)}, f x_{n(k)-1}\right) \\
= & G\left(f x_{m(k)}, f x_{m(k)-1}, f x_{n(k)}\right) \\
& +G_{n(k)-1}, \quad \forall k \in N,
\end{aligned}
$$

$$
\begin{aligned}
G\left(f x_{m(k)}, f x_{m(k)-1}, f x_{n(k)}\right)= & G\left(f x_{n(k)}, f x_{m(k)-1}, f x_{m(k)}\right) \\
\leq & G\left(f x_{n(k)}, f x_{m(k)}, f x_{m(k)}\right) \\
& +G\left(f x_{m(k)}, f x_{m(k)-1}, f x_{m(k)}\right) \\
\leq & G\left(f x_{n(k)}, f x_{n(k)-1}, f x_{m(k)}\right) \\
& +G\left(f x_{m(k)}, f x_{m(k)-1}, f x_{m(k)}\right) \\
\leq & G\left(f x_{n(k)}, f x_{n(k)-1}, f x_{n(k)-1}\right) \\
& +G\left(f x_{n(k)-1}, f x_{n(k)-1}, f x_{m(k)}\right) \\
& +G\left(f x_{m(k)}, f x_{m(k)-1}, f x_{m(k)}\right) \\
\leq & G_{n(k)-1}+2 G_{m(k)-1} \\
& +G\left(f x_{n(k)-1}, f x_{n(k)-1}, f x_{m(k)}\right), \quad \forall k \in \mathbb{N} .
\end{aligned}
$$

Taking $k \rightarrow \infty$ in (34) and (35) and utilizing (23) and (33), we conclude that

$$
\varepsilon=\lim _{k \rightarrow \infty} G\left(f x_{n(k)-1}, f x_{n(k)-1}, f x_{m(k)}\right) .
$$

On the basis of (G3)-(G5) and Lemma 13, we arrive at

$$
\begin{aligned}
G\left(f x_{n(k)-1}, f x_{m(k)}, f x_{n(k)}\right)= & G\left(f x_{n(k)}, f x_{n(k)-1}, f x_{m(k)}\right) \\
\leq & G\left(f x_{n(k)}, f x_{n(k)-1}, f x_{n(k)-1}\right) \\
& +G\left(f x_{n(k)-1}, f x_{n(k)-1}, f x_{m(k)}\right) \\
\leq & G_{n(k)-1}+G \\
& \cdot\left(f x_{n(k)-1}, f x_{n(k)-1}, f x_{m(k)}\right), \quad \forall k \in \mathbb{N},
\end{aligned}
$$

$$
\begin{aligned}
G\left(f x_{n(k)-1}, f_{x_{n(k)-1}}, f x_{m(k)}\right) \leq & G\left(f x_{n(k)-1}, f x_{n(k)}, f x_{n(k)}\right) \\
& +G\left(f x_{n(k)}, f x_{n(k)-1}, f x_{m(k)}\right) \\
\leq & 2 G\left(f x_{n(k)}, f x_{n(k)-1}, f x_{n(k)-1}\right) \\
& +G\left(f x_{n(k)-1}, f x_{m(k)}, f x_{n(k)}\right) \\
\leq & 2 G_{n(k)-1}+G\left(f x_{n(k)-1}, f x_{m(k)}, f x_{n(k)}\right), \quad \forall k \in \mathbb{N} .
\end{aligned}
$$

Letting $k \rightarrow \infty$ in (37) and (38) and using (23) and (36), we deduce that

$$
\varepsilon=\lim _{k \rightarrow \infty} G\left(f x_{n(k)-1}, f x_{m(k)}, f x_{n(k)}\right) .
$$

On account of (G3)-(G5) and Lemma 13, we receive that

$$
\begin{aligned}
G\left(f x_{n(k)}, f x_{n(k)}, f x_{m(k)-1}\right)= & G\left(f x_{m(k)-1}, f x_{n(k)}, f x_{n(k)}\right) \\
\leq & G\left(f x_{m(k)-1}, f x_{n(k)-1}, f x_{n(k)-1}\right) \\
& +G\left(f x_{n(k)-1}, f x_{n(k)}, f x_{n(k)}\right) \\
\leq & G\left(f x_{n(k)-1}, f x_{m(k)-1}, f x_{m(k)}\right) \\
& +2 G_{n(k)-1}, \quad \forall k \in \mathbb{N},
\end{aligned}
$$

$$
\begin{aligned}
G\left(f x_{n(k)-1}, f x_{m(k)-1}, f x_{m(k)}\right) \leq & G\left(f x_{n(k)-1}, f x_{m(k)-1}, f x_{m(k)-1}\right) \\
& +G\left(f x_{m(k)-1}, f x_{m(k)-1}, f x_{m(k)}\right) \\
\leq & G\left(f x_{n(k)-1}, f x_{n(k)}, f x_{m(k)-1}\right) \\
& +G_{m(k)-1} \leq G\left(f x_{n(k)-1}, f x_{n(k)}, f x_{n(k)}\right) \\
& +G\left(f x_{n(k)}, f x_{n(k)}, f x_{m(k)-1}\right)+G_{m(k)-1} \\
& +\leq 2 G_{n(k)-1}+G\left(f x_{n(k)}, f x_{n(k)}, f x_{m(k)-1}\right) \\
& +G_{m(k)-1}, \quad \forall k \in \mathbb{N} .
\end{aligned}
$$

Taking $k \rightarrow \infty$ in (40) and (41) and using (23) and (33), we conclude that

$$
\varepsilon=\lim _{k \rightarrow \infty} G\left(f x_{n(k)}, f x_{n(k)}, f x_{m(k)-1}\right) .
$$

Making use of (12)-(14), (23), (25), (33), (36), (39), (42), $(\varphi, \psi, \phi) \in \Phi_{1} \times \Phi_{2} \times \Phi_{3}$, and Lemma 12 , we obtain that 


$$
\begin{aligned}
& \lim _{k \rightarrow \infty} M_{1}\left(x_{m(k)}, x_{m(k)}, x_{n(k)}\right) \\
& =\lim _{k \rightarrow \infty} \max \left\{G\left(g x_{m(k)}, g x_{m(k)}, g x_{n(k)}\right),\right. \\
& \frac{\left[1+G\left(g x_{m(k)}, g x_{m(k)}, f x_{m(k)}\right)\right] G\left(g x_{m(k)}, g x_{m(k)}, f x_{n(k)}\right)}{2+G\left(g x_{m(k)}, f x_{m(k)}, f x_{m(k)}\right)}, \\
& \frac{\left[1+G\left(g x_{m(k)}, g x_{m(k)}, f x_{m(k)}\right)\right] G\left(g x_{n(k)}, g x_{n(k)}, f x_{m(k)}\right)}{2+G\left(g x_{m(k)}, f x_{m(k)}, f x_{n(k)}\right)}, \\
& \frac{\left[1+G\left(g x_{m(k)}, g x_{m(k)}, f x_{m(k)}\right)\right] G\left(g x_{n(k)}, g x_{n(k)}, f x_{m(k)}\right)}{2+G\left(g x_{m(k)}, f x_{m(k)}, f x_{n(k)}\right)}, \\
& \frac{\left[1+G\left(g x_{m(k)}, g x_{m(k)}, f x_{m(k)}\right)\right] G\left(g x_{m(k)}, g x_{m(k)}, f x_{n(k)}\right)}{2\left[1+G\left(g x_{m(k)}, f x_{m(k)}, f x_{m(k)}\right)\right]}, \\
& \frac{\left[1+G\left(g x_{n(k)}, g x_{n(k)}, f x_{n(k)}\right)\right] G\left(g x_{m(k)}, g x_{m(k)}, f x_{m(k)}\right)}{2\left[1+G\left(g x_{n(k)}, f x_{m(k)}, f x_{n(k)}\right)\right]}, \\
& \frac{\left[1+G\left(g x_{n(k)}, g x_{n(k)}, f x_{n(k)}\right)\right] G\left(g x_{m(k)}, g x_{m(k)}, f x_{m(k)}\right)}{2\left[1+G\left(g x_{n(k)}, f x_{m(k)}, f x_{n(k)}\right)\right]}, \\
& \frac{\left[1+G\left(g x_{m(k)}, g x_{m(k)}, g x_{n(k)}\right)\right] G\left(f x_{m(k)}, f x_{m(k)}, f x_{n(k)}\right)}{1+G\left(g x_{n(k)}, f x_{m(k)}, f x_{m(k)}\right)+G\left(g x_{m(k)}, g x_{m(k)}, g x_{n(k)}\right)}, \\
& \overline{1+G\left(g x_{n(k)}, f x_{m(k)}, f x_{m(k)}\right)+G\left(g x_{m(k)}, g x_{m(k)}, g x_{n(k)}\right)} \\
& \underline{\left[1+G\left(g x_{m(k)}, g x_{m(k)}, g x_{n(k)}\right)\right] G\left(f x_{m(k)}, f x_{m(k)}, f x_{n(k)}\right)} \\
& \overline{1+G\left(g x_{m(k)}, f x_{m(k)}, f x_{m(k)}\right)+G\left(g x_{m(k)}, g x_{m(k)}, g x_{n(k)}\right)} \\
& \left.\frac{\left[1+G\left(g x_{m(k)}, g x_{m(k)}, g x_{n(k)}\right)\right] G\left(f x_{m(k)}, f x_{m(k)}, f x_{n(k)}\right)}{1+G\left(g x_{m(k)}, f x_{n(k)}, f x_{n(k)}\right)+G\left(g x_{m(k)}, g x_{m(k)}, g x_{\mathrm{n}(k)}\right)}\right\} \\
& =\lim _{k \rightarrow \infty} \max \left\{G\left(f x_{m(k)-1}, f x_{m(k)-1}, f x_{n(k)-1}\right)\right. \text {, } \\
& \frac{\left[1+G\left(f x_{m(k)-1}, f x_{m(k)-1}, f x_{m(k)}\right)\right] G\left(f x_{m(k)-1}, f x_{m(k)-1}, f x_{n(k)}\right)}{2+G\left(f x_{m(k)-1}, f x_{m(k)}, f x_{m(k)}\right)}, \\
& \frac{\left[1+G\left(f x_{m(k)-1}, f x_{m(k)-1}, f x_{m(k)}\right)\right] G\left(f x_{n(k)-1}, f x_{n(k)-1}, f x_{m(k)}\right)}{2+G\left(f x_{m(k)-1}, f x_{m(k)}, f x_{n(k)}\right)}, \\
& \frac{\left[1+G\left(f x_{m(k)-1}, f x_{m(k)-1}, f x_{m(k)}\right)\right] G\left(f x_{n(k)-1}, f x_{n(k)-1}, f x_{m(k)}\right)}{2+G\left(f x_{m(k)-1}, f x_{m(k)}, f x_{n(k)}\right)}, \\
& \frac{\left[1+G\left(f x_{m(k)-1}, f x_{m(k)-1}, f x_{m(k)}\right)\right] G\left(f x_{m(k)-1}, f x_{m(k)-1}, f x_{n(k)}\right)}{2\left[1+G\left(f x_{m(k)-1}, f x_{m(k)}, f x_{m(k)}\right)\right]}, \\
& \frac{\left[1+G\left(f x_{n(k)-1}, f x_{n(k)-1}, f x_{n(k)}\right)\right] G\left(f x_{m(k)-1}, f x_{m(k)-1}, f x_{m(k)}\right)}{2\left[1+G\left(f x_{n(k)-1}, f x_{m(k)}, f x_{n(k)}\right)\right]}, \\
& \frac{\left[1+G\left(f x_{n(k)-1}, f x_{n(k)-1}, f x_{n(k)}\right)\right] G\left(f x_{m(k)-1}, f x_{m(k)-1}, f x_{m(k)}\right)}{2\left[1+G\left(f x_{n(k)-1}, f x_{m(k)}, f x_{n(k)}\right)\right]}, \\
& \frac{\left[1+G\left(f x_{m(k)-1}, f x_{m(k)-1}, f x_{n(k)-1}\right)\right] G\left(f x_{m(k)}, f x_{m(k)}, f x_{n(k)}\right)}{1+G\left(f x_{n(k)-1}, f x_{m(k)}, f x_{m(k)}\right)+G\left(f x_{m(k)-1}, f x_{m(k)-1}, f x_{n(k)-1}\right)}, \\
& \frac{\left[1+G\left(f x_{m(k)-1}, f x_{m(k)-1}, f x_{n(k)-1}\right)\right] G\left(f x_{m(k)}, f x_{m(k)}, f x_{n(k)}\right)}{1+G\left(f x_{m(k)-1}, f x_{m(k)}, f x_{m(k)}\right)+G\left(f x_{m(k)-1}, f x_{m(k)-1}, f x_{n(k)-1}\right)},
\end{aligned}
$$

$$
\begin{gathered}
\left.\frac{\left[1+G\left(f x_{m(k)-1}, f x_{m(k)-1}, f x_{n(k)-1}\right)\right] G\left(f x_{m(k)}, f x_{m(k)}, f x_{n(k)}\right)}{1+G\left(f x_{m(k)-1}, f x_{n(k)}, f x_{n(k)}\right)+G\left(f x_{m(k)-1}, f x_{m(k)-1}, f x_{n(k)-1}\right)}\right\} \\
\quad=\max \left\{\varepsilon, \frac{\varepsilon}{2}, \frac{\varepsilon}{2+\varepsilon}, \frac{\varepsilon}{2+\varepsilon}, \frac{\varepsilon}{2}, 0,0, \frac{(1+\varepsilon) \varepsilon}{1+2 \varepsilon}, \varepsilon, \frac{(1+\varepsilon) \varepsilon}{1+2 \varepsilon}\right\}=\varepsilon
\end{gathered}
$$

Making use of (12), (33), (43), $(\varphi, \psi, \phi) \in \Phi_{1} \times \Phi_{2} \times \Phi_{3}$, and Lemma 17, we conclude that

$$
\begin{aligned}
\psi\left(\int_{0}^{\varepsilon} \varphi(t) d t\right)= & \limsup _{k \rightarrow \infty} \psi\left(\int_{0}^{G\left(f x_{m(k)}, f x_{m(k)}, f x_{n(k)}\right)} \varphi(t) d t\right) \\
\leq & \limsup _{k \rightarrow \infty}\left[\psi\left(\int_{0}^{M_{1}\left(x_{m(k)}, x_{m(k)}, x_{n(k)}\right)} \varphi(t) d t\right)\right. \\
& \left.-\phi\left(\int_{0}^{M_{1}\left(x_{m(k)}, x_{m(k)}, x_{n(k)}\right)} \varphi(t) d t\right)\right] \\
\leq & \limsup _{k \rightarrow \infty}\left(\int_{0}^{M_{1}\left(x_{m(k)}, x_{m(k)}, x_{n(k)}\right)} \varphi(t) d t\right) \\
& -\lim _{k \rightarrow \infty} \inf \phi\left(\int_{0}^{M_{1}\left(x_{m(k)}, x_{m(k)}, x_{n(k)}\right)} \varphi(t) d t\right) \\
= & \left.\psi\left(\int_{0}^{\varepsilon} \varphi(t) d t\right)-\liminf _{k \rightarrow \infty} \sin ^{\varepsilon}\right) \\
& \cdot\left(\int_{0}^{M_{1}\left(x_{m(k)}, x_{m(k)}, x_{n(k)}\right)} \varphi(t) d t\right) \\
< & \left(\int_{0}^{\varepsilon} \varphi(t) d t\right),
\end{aligned}
$$

which is ridiculous. Thus, $\left\{f x_{n}\right\}_{n \in \mathbb{N}_{0}}$ is a $G$-Cauchy sequence. Since $g(X)$ is complete, it follows that there exists $w \in g(X)$ such that

$$
\lim _{n \rightarrow \infty} f x_{n}=w
$$

In light of Lemma 8 and $w \in g(X)$, there exists $a \in X$ satisfying $g a=w$ and

$$
\lim _{n \rightarrow \infty} G\left(f x_{n}, f x_{n}, g a\right)=\lim _{n \rightarrow \infty} G\left(f x_{n}, g a, g a\right)=0 .
$$

Next, we prove $g a=f a$. Suppose that $g a \neq f a$. In view of (12), (13), (25), (46), $(\varphi, \psi, \phi) \in \Phi_{1} \times \Phi_{2} \times \Phi_{3}$, and Lemmas 12 and 17 , we obtain that 
$\lim _{n \rightarrow \infty} M_{1}\left(x_{n}, x_{n}, a\right)=\lim _{n \rightarrow \infty} \max \left\{G\left(g x_{n}, g x_{n}, g a\right)\right.$,

$$
\begin{aligned}
& \frac{\left[1+G\left(g x_{n}, g x_{n}, f x_{n}\right)\right] G\left(g x_{n}, g x_{n}, f a\right)}{2+G\left(g x_{n}, f x_{n}, f x_{n}\right)}, \\
& \frac{\left[1+G\left(g x_{n}, g x_{n}, f x_{n}\right)\right] G\left(g a, g a, f x_{n}\right)}{2+G\left(g x_{n}, f x_{n}, f a\right)}, \\
& 2+G\left(g x_{n}, f x_{n}, f a\right) \\
& \frac{\left[1+G\left(g x_{n}, g x_{n}, f x_{n}\right)\right] G\left(g a, g a, f x_{n}\right)}{2+G\left(g x_{n}, f x_{n}, f a\right)}, \\
& \frac{\left[1+G\left(g x_{n}, g x_{n}, f x_{n}\right)\right] G\left(g x_{n}, g x_{n}, f a\right)}{2\left[1+G\left(g x_{n}, f x_{n}, f x_{n}\right)\right]}, \\
& \frac{[1+G(g a, g a, f a)] G\left(g x_{n}, g x_{n}, f x_{n}\right)}{2\left[1+G\left(g a, f x_{n} f a\right)\right]}, \\
& 2\left[1+G\left(g a, f x_{n}, f a\right)\right. \\
& \underline{[1+G(g a, g a, f a)] G\left(g x_{n}, g x_{n}, f x_{n}\right)}, \\
& 2\left[1+G\left(g a, f x_{n}, f a\right)\right] \\
& {\left[1+G\left(g x_{n}, g x_{n}, g a\right)\right] G\left(f x_{n}, f x_{n}, f a\right)} \\
& 1+G\left(g a, f x_{n}, f x_{n}\right)+G\left(g x_{\mathrm{n}}, g x_{n}, g a\right) \text {, } \\
& {\left[1+G\left(g x_{n}, g x_{n}, g a\right)\right] G\left(f x_{n}, f x_{n}, f a\right)} \\
& \overline{1+G\left(g x_{n}, f x_{n}, f x_{n}\right)+G\left(g x_{n}, g x_{n}, g a\right)}, \\
& \left.\underline{\left[1+G\left(g x_{n}, g x_{n}, g a\right)\right] G\left(f x_{n}, f x_{n}, f a\right)}\right\} \\
& \left.\overline{1+G\left(g x_{n}, f a, f a\right)+G\left(g x_{n}, g x_{n}, g a\right)}\right\} \\
& =\lim _{n \rightarrow \infty} \max \left\{G\left(f x_{n-1}, f x_{n-1}, g a\right)\right. \text {, } \\
& \frac{\left[1+G\left(f x_{n-1}, f x_{n-1}, f x_{n}\right)\right] G\left(f x_{n-1}, f x_{n-1}, f a\right)}{2+G\left(f x_{n-1}, f x_{n}, f x_{n}\right)}, \\
& \frac{\left[1+G\left(f x_{n-1}, f x_{n-1}, f x_{n}\right)\right] G\left(g a, g a, f x_{n}\right)}{2+G\left(f x_{n-1}, f x_{n}, f a\right)}, \\
& \frac{\left[1+G\left(f x_{n-1}, f x_{n-1}, f x_{n}\right)\right] G\left(g a, g a, f x_{n}\right)}{2+G\left(f x_{n-1}, f x_{n}, f a\right)}, \\
& \frac{\left[1+G\left(f x_{n-1}, f x_{n-1}, f x_{n}\right)\right] G\left(f x_{n-1}, f x_{n-1}, f a\right)}{2\left[1+G\left(f x_{n-1}, f x_{n}, f x_{n}\right)\right]}, \\
& \frac{[1+G(g a, g a, f a)] G\left(f x_{n-1}, f x_{n-1}, f x_{n}\right)}{2\left[1+G\left(g a, f x_{n}, f a\right)\right]}, \\
& \frac{[1+G(g a, g a, f a)] G\left(f x_{n-1}, f x_{n-1}, f x_{n}\right)}{2\left[1+G\left(g a, f x_{n}, f a\right)\right]} \\
& 2\left[1+G\left(g a, f x_{n}, f a\right)\right] \\
& {\left[1+G\left(f x_{n-1}, f x_{n-1}, g a\right)\right] G\left(f x_{n}, f x_{n}, f a\right)} \\
& \overline{1+G\left(g a, f x_{n}, f x_{n}\right)+G\left(f x_{n-1}, f x_{n-1}, g a\right)}, \\
& \frac{\left[1+G\left(f x_{n-1}, f x_{n-1}, g a\right)\right] G\left(f x_{n}, f x_{n}, f a\right)}{1+G\left(f x_{n-1}, f x_{n}, f x_{n}\right)+G\left(f x_{n-1}, f x_{n-1}, g a\right)} \\
& \left.\frac{\left[1+G\left(f x_{n-1}, f x_{n-1}, g a\right)\right] G\left(f x_{n}, f x_{n}, f a\right)}{1+G\left(f x_{n-1}, f a, f a\right)+G\left(f x_{n-1}, f x_{n-1}, g a\right)}\right\}
\end{aligned}
$$

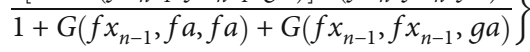

$$
\begin{aligned}
& =\max \left\{0, \frac{G(g a, g a, f a)}{2}, 0,0\right. \text {, } \\
& \frac{G(g a, g a, f a)}{2}, 0,0, G(g a, g a, f a), G(g a, g a, f a) \\
& \left.\frac{G(g a, g a, f a)}{1+G(g a, f a, f a)}\right\}=G(g a, g a, f a)
\end{aligned}
$$

$$
\begin{aligned}
\psi\left(\int_{0}^{G(g a, g a, f a)} \varphi(t) d t\right)= & \limsup _{n \rightarrow \infty} \psi\left(\int_{0}^{G\left(f x_{n}, f x_{n}, f a\right)} \varphi(t) d t\right) \\
\leq & \limsup _{n \rightarrow \infty}\left[\psi\left(\int_{0}^{M_{1}\left(x_{n}, x_{n}, a\right)} \varphi(t) d t\right)\right. \\
& \left.-\phi\left(\int_{0}^{M_{1}\left(x_{n}, x_{n}, a\right)} \varphi(t) d t\right)\right]
\end{aligned}
$$

$$
\begin{aligned}
& \leq \limsup _{n \rightarrow \infty} \psi\left(\int_{0}^{M_{1}\left(x_{n}, x_{n}, a\right)} \varphi(t) d t\right)-\liminf _{n \rightarrow \infty} \phi\left(\int_{0}^{M_{1}\left(x_{n}, x_{n}, a\right)} \varphi(t) d t\right) \\
& \quad=\psi\left(\int_{0}^{G(g a, g a, f a)} \varphi(t) d t\right)-\liminf _{n \rightarrow \infty} \phi\left(\int_{0}^{M_{1}\left(x_{n}, x_{n}, a\right)} \varphi(t) d t\right) \\
& \quad<\psi\left(\int_{0}^{G(g a, g a, f a)} \varphi(t) d t\right),
\end{aligned}
$$

which is absurd. Consequently, $w=g a=f a$, that is, $w$ is a point of coincidence of $f$ and $g$.

Lastly, we certify that $f$ and $g$ have a unique point of coincidence in $X$. Assume that there exists $b \in X$ with $f b=$ $g b \neq f a$. In terms of (13), (G2), and Lemma 13, we receive that

$$
\begin{aligned}
M_{1}(a, a, b)= & \max \left\{G(g a, g a, g b), \frac{[1+G(g a, g a, f a)] G(g a, g a, f b)}{2+G(g a, f a, f a)},\right. \\
& \frac{[1+G(g a, g a, f a)] G(g b, g b, f a)}{2+G(g a, f a, f b)}, \frac{[1+G(g a, g a, f a)] G(g b, g b, f a)}{2+G(g a, f a, f b)}, \\
& \frac{[1+G(g a, g a, f a)] G(g a, g a, f b)}{2[1+G(g a, f a, f a)]}, \frac{[1+G(g b, g b, f b)] G(g a, g a, f a)}{2[1+G(g b, f a, f b)]}, \\
& \frac{[1+G(g b, g b, f b)] G(g a, g a, f a)}{2[1+G(g b, f a, f b)]}, \frac{[1+G(g a, g a, g b)] G(f a, f a, f b)}{1+G(g b, f a, f a)+G(g a, g a, g b)}, \\
& \left.\frac{[1+G(g a, g a, g b)] G(f a, f a, f b)}{1+G(g a, f a, f a)+G(g a, g a, g b)}, \frac{[1+G(g a, g a, g b)] G(f a, f a, f b)}{1+G(g a, f b, f b)+G(g a, g a, g b)}\right\} \\
= & \max \left\{G(f a, f a, f b), \frac{G(f a, f a, f b)}{2}, \frac{G(f b, f b, f a)}{2+G(f a, f a, f b)},\right. \\
& \frac{G(f b, f b, f a)}{2+G(f a, f a, f b)}, \frac{G(f a, f a, f b)}{2}, 0,0, \\
& \frac{[1+G(f a, f a, f b)] G(f a, f a, f b)}{1+2 G(f a, f a, f b)}, \quad G(f a, f a, f b), \\
& \left.\frac{[1+G(f a, f a, f b)] G(f a, f a, f b)}{1+G(f a, f b, f b)+G(f a, f a, f b)}\right\}=G(f a, f a, f b)>0 .
\end{aligned}
$$

According to (12), (49), $(\varphi, \psi, \phi) \in \Phi_{1} \times \Phi_{2} \times \Phi_{3}$, and Lemma 18 , we gain that

$$
\begin{aligned}
\psi\left(\int_{0}^{G(f a, f a, f b)} \varphi(t) d t\right) & \leq \psi\left(\int_{0}^{M_{1}(a, a, b)} \varphi(t) d t\right)-\phi\left(\int_{0}^{M_{1}(a, a, b)} \varphi(t) d t\right) \\
& =\psi\left(\int_{0}^{G(f a, f a, f b)} \varphi(t) d t\right)-\phi\left(\int_{0}^{G(f a, f a, f b)} \varphi(t) d t\right) \\
& <\psi\left(\int_{0}^{G(f a, f a, f b)} \varphi(t) d t\right)
\end{aligned}
$$

which is contradictive. Therefore, $f$ and $g$ have a unique point of coincidence in $X$. Moreover, if $f$ and $g$ are weakly compatible mappings, by Lemma 16, we know that $f$ and $g$ have a unique common fixed point in $X$. This completes the proof.

Similar to the argument of Theorem 19, we derive the following result and omit its proof. 
Theorem 20. Let $(X, G)$ be a $G$-metric space, $f$ and $g: X \rightarrow X$ be two mappings satisfying

$\psi\left(\int_{0}^{G(f x, f y, f z)} \varphi(t) d t\right) \leq \psi\left(\int_{0}^{M_{2}(x, y, z)} \varphi(t) d t\right)-\phi\left(\int_{0}^{M_{2}(x, y, z)} \varphi(t) d t\right), \quad \forall x, y, z \in X$,

where $(\varphi, \psi, \phi) \in \Phi_{1} \times \Phi_{2} \times \Phi_{3}$ and

$$
\begin{aligned}
M_{2}(x, y, z)= & \max \left\{G(g x, g y, g z), \frac{[1+G(g x, f x, f x)] G(g z, f y, f y)}{2+G(g x, g y, f x)},\right. \\
& \frac{[1+G(g y, f y, f y)] G(g z, f x, f x)}{2+G(g x, g y, f y)}, \frac{[1+G(g z, f z, f z)] G(g x, f y, f y)}{2+G(g y, g z, f z)}, \\
& \frac{[1+G(g x, f x, f x)] G(g y, f z, f z)}{2[1+G(g x, g z, f x)]}, \frac{[1+G(g y, f y, f y)] G(g x, f z, f z)}{2[1+G(g y, g z, f y)]}, \\
& \frac{[1+G(g z, f z, f z)] G(g y, f x, f x)}{2[1+G(g x, g z, f z)]}, \frac{[1+G(g x, g y, g z)] G(f x, f y, f z)}{1+G(g z, g z, f y)+G(g x, g y, g z)}, \\
& \left.\frac{[1+G(g x, g y, g z)] G(f x, f y, f z)}{1+G(g y, g y, f x)+G(g x, g y, g z)}, \frac{[1+G(g x, g y, g z)] G(f x, f y, f z)}{1+G(g x, g x, f z)+G(g x, g y, g z)}\right\} .
\end{aligned}
$$

If $f(X) \subseteq g(X)$ and $g(X)$ is a complete subset of $X$, then $f$ and $g$ have a unique point of coincidence in $X$. Furthermore, if $f$ and $g$ are weakly compatible mappings, then $f$ and $g$ have a unique common fixed point in $X$.

Remark 21. In case $\psi(t)=t, \phi(t)=(1-\lambda) t, \forall t \in R^{+}$and $\lambda \epsilon$ $(0,1)$ is a constant, then Theorems 19 and 20 reduce to results, which include Theorem 5 as a special case. The following example shows that Theorems 19 and 20 generalize substantially Theorem 5 and differ from Theorem 4 .

Example 22. Let $X=[0,2]$. Define $f, g: X \rightarrow X, \varphi, \psi, \phi, \eta$ $: \mathbb{R}^{+} \rightarrow \mathbb{R}^{+}$, and $G: X \times X \times X \rightarrow R^{+}$by

$$
\begin{gathered}
f x=\left\{\begin{array}{l}
0, \quad \forall x \in[0,1], \\
\frac{1}{6}, \quad \forall x \in(1,2],
\end{array} \quad g x= \begin{cases}x, & \forall x \in[0,1], \\
\frac{7}{6}, & \forall x \in(1,2],\end{cases} \right. \\
\varphi(t)=2 t, \psi(t)=3 t, \phi(t)=t, \eta(t)=2 t, \quad \forall t \in \mathbb{R}^{+}, \\
G(x, y, z)=|x-y|+|y-z|+|z-x|, \forall x, y, z \in X .
\end{gathered}
$$

Clearly, $(X, G)$ is a $G$-metric space, $(\varphi, \psi, \phi) \in \Phi_{1} \times \Phi_{2}$ $\times \Phi_{3}, f$ and $g$ are weakly compatible mappings, $f(X) \subseteq g($ $X)$ and $g(X)$ is complete, $\eta(t)=\psi(t)-\phi(t), \forall t \in \mathbb{R}^{+}$and $\eta$ is nondecreasing in $\mathbb{R}^{+}$. Let $x, y, z \in X$. In order to verify (12) and (51), we consider the following seven cases:

Case 1. $x, y, z \in[0,1]$ or $x, y, z \in(1,2]$. It follows that

$$
\begin{aligned}
\psi\left(\int_{0}^{G(f x, f y, f z)} \varphi(t) d t\right)= & \leq \leq \eta\left(\int_{0}^{M_{i}(x, y, z)} \varphi(t) d t\right)=\psi\left(\int_{0}^{M_{i}(x, y, z)} \varphi(t) d t\right) \\
& -\phi\left(\int_{0}^{M_{i}(x, y, z)} \varphi(t) d t\right), \quad \forall i \in\{1,2\} .
\end{aligned}
$$

Case 2. $x, y \in[0,1]$ and $z \in(1,2]$. It follows that

$$
\begin{aligned}
M_{1}(x, y, z) \geq & \frac{[1+G(g y, g y, f y)] G(g z, g z, f x)}{2+G(g y, f y, f z)} \\
& =\frac{[1+G(y, y, 0)] G(7 / 6,7 / 6,0)}{2+G(y, 0,1 / 6)} \\
& =\frac{(7 / 3)(1+2 y)}{2+y+1 / 6+|y-1 / 6|} \geq 1, \\
M_{2}(x, y, z) \geq & \frac{[1+G(g y, f y, f y)] G(g z, f x, f x)}{2+G(g x, g y, f y)} \\
= & \frac{[1+G(y, 0,0)] G(7 / 6,0,0)}{2+G(x, y, 0)} \\
= & \frac{(7 / 3)(1+2 y)}{2+x+y+|x-y|} \geq \frac{7}{12} .
\end{aligned}
$$

It is easy to see that

$$
\begin{aligned}
& M_{i}(x, y, z) \geq \frac{7}{12} \\
& \psi\left(\int_{0}^{G(f x, f y, f z)} \varphi(t) d t\right)= 3 \int_{0}^{1 / 3} 2 t d t=\frac{1}{3}<2 \cdot \frac{49}{144} \\
&= \eta\left(\int_{0}^{7 / 12} \varphi(t) d t\right) \leq \eta\left(\int_{0}^{M_{i}(x, y, z)} \varphi(t) d t\right) \\
&= \psi\left(\int_{0}^{M_{i}(x, y, z)} \varphi(t) d t\right) \\
&-\phi\left(\int_{0}^{M_{i}(x, y, z)} \varphi(t) d t\right), \quad \forall i \in\{1,2\} .
\end{aligned}
$$

Case 3. $x, z \in[0,1]$ and $y \in(1,2]$. It follows that

$$
\begin{aligned}
& M_{1}(x, y, z) \geq \frac{[1+G(g z, g z, f z)] G(g y, g y, f x)}{2[1+G(g z, f y, f z)]} \\
& =\frac{[1+G(z, z, 0)] G(7 / 6,7 / 6,0)}{2[1+G(z, 1 / 6,0)]} \\
& =\frac{(7 / 3)(1+2 z)}{2(1+1 / 6+z+|z-1 / 6|)} \geq \frac{7}{8} \text {, } \\
& M_{2}(x, y, z) \geq \max \left\{\frac{[1+G(g x, f x, f x)] G(g y, f z, f z)}{2[1+G(g x, g z, f x)]},\right. \\
& \left.\frac{[1+G(g z, f z, f z)] G(g y, f x, f x)}{2[1+G(g x, g z, f z)]}\right\} \\
& =\max \left\{\frac{[1+G(x, 0,0)] G(7 / 6,0,0)}{2[1+G(x, z, 0)]},\right. \\
& \left.\frac{[1+G(z, 0,0)] G(7 / 6,0,0)}{2[1+G(x, z, 0)]}\right\} \\
& =\max \left\{\frac{(7 / 3)(1+2 x)}{2(1+x+z+|x-z|)}, \frac{(7 / 3)(1+2 z)}{2(1+x+z+|x-z|)}\right\} \\
& \geq \frac{7}{6}
\end{aligned}
$$


It is obvious that

$$
\begin{aligned}
& M_{i}(x, y, z) \geq \frac{7}{8} \\
& \psi\left(\int_{0}^{G(f x, f y, f z)} \varphi(t) d t\right)= 3 \int_{0}^{1 / 3} 2 t d t=\frac{1}{3}<2 \cdot \frac{49}{64} \\
&= \eta\left(\int_{0}^{7 / 8} \varphi(t) d t\right) \leq \eta\left(\int_{0}^{M_{i}(x, y, z)} \varphi(t) d t\right) \\
&= \psi\left(\int_{0}^{M_{i}(x, y, z)} \varphi(t) d t\right) \\
&-\phi\left(\int_{0}^{M_{i}(x, y, z)} \varphi(t) d t\right), \quad \forall i \in\{1,2\} .
\end{aligned}
$$

Case 4. $y, z \in[0,1]$ and $x \in(1,2]$. It follows that

$$
\begin{gathered}
M_{1}(x, y, z) \geq \frac{[1+G(g y, g y, f y)] G(g x, g x, f z)}{2[1+G(g y, f x, f y)]} \\
=\frac{[1+G(y, y, 0)] G(7 / 6,7 / 6,0)}{2[1+G(y, 1 / 6,0)]} \\
=\frac{(7 / 3)(1+2 y)}{2(1+y+1 / 6+|y-1 / 6|)} \geq \frac{7}{8}, \\
M_{2}(x, y, z) \geq \frac{[1+G(g z, f z, f z)] G(g x, f y, f y)}{2+G(g y, g z, f z)} \\
=\frac{[1+G(z, 0,0)] G(7 / 6,0,0)}{2+G(y, z, 0)}=\frac{(7 / 3)(1+2 z)}{2+y+z+|y-z|} \\
\geq \frac{7}{12} .
\end{gathered}
$$

It is apparent that

$$
\begin{aligned}
M_{i}(x, y, z) \geq \frac{7}{12} & \\
\psi\left(\int_{0}^{G(f x, f y, f z)} \varphi(t) d t\right)= & 3 \int_{0}^{1 / 3} 2 t d t=\frac{1}{3}<2 \cdot \frac{49}{144} \\
= & \eta\left(\int_{0}^{7 / 12} \varphi(t) d t\right) \leq \eta\left(\int_{0}^{M_{i}(x, y, z)} \varphi(t) d t\right) \\
= & \psi\left(\int_{0}^{M_{i}(x, y, z)} \varphi(t) d t\right) \\
& -\phi\left(\int_{0}^{M_{i}(x, y, z)} \varphi(t) d t\right), \quad \forall i \in\{1,2\} .
\end{aligned}
$$

Case 5. $x \in[0,1]$ and $y, z \in(1,2]$. It follows that

$$
\begin{aligned}
M_{1}(x, y, z) & \geq \frac{[1+G(g z, g z, f z)] G(g y, g y, f x)}{2[1+G(g z, f y, f z)]} \\
& =\frac{[1+G(7 / 6,7 / 6,1 / 6)] G(7 / 6,7 / 6,0)}{2[1+G(7 / 6,1 / 6,1 / 6)]} \\
& =\frac{(7 / 3)(1+2)}{2(1+2)}=\frac{7}{6}, \\
M_{2}(x, y, z) \geq & \frac{[1+G(g y, f y, f y)] G(g z, f x, f x)}{2+G(g x, g y, f y)} \\
& =\frac{[1+G(7 / 6,1 / 6,1 / 6)] G(7 / 6,0,0)}{2+G(x, 7 / 6,1 / 6)} \\
& =\frac{(7 / 3)(1+2)}{2+1+|x-7 / 6|+|x-1 / 6|} \geq \frac{21}{13} .
\end{aligned}
$$

It is distinct that

$$
\begin{aligned}
& M_{i}(x, y, z) \geq \frac{7}{6} \\
& \psi\left(\int_{0}^{G(f x, f y, f z)} \varphi(t) d t\right)= 3 \int_{0}^{1 / 3} 2 t d t=\frac{1}{3}<2 \cdot \frac{49}{36} \\
&= \eta\left(\int_{0}^{7 / 6} \varphi(t) d t\right) \leq \eta\left(\int_{0}^{M_{i}(x, y, z)} \varphi(t) d t\right) \\
&= \psi\left(\int_{0}^{M_{i}(x, y, z)} \varphi(t) d t\right) \\
&-\phi\left(\int_{0}^{M_{i}(x, y, z)} \varphi(t) d t\right), \quad \forall i \in\{1,2\} .
\end{aligned}
$$

Case 6. $y \in[0,1]$ and $x, z \in(1,2]$. It follows that

$$
\begin{aligned}
M_{1}(x, y, z) & \geq \frac{[1+G(g y, g y, f y)] G(g x, g x, f z)}{2[1+G(g y, f x, f y)]} \\
& =\frac{[1+G(y, y, 0)] G(7 / 6,7 / 6,1 / 6)}{2[1+G(y, 1 / 6,0)]} \\
& =\frac{2(1+2 y)}{2(1+y+1 / 6+|y-1 / 6|)} \geq \frac{3}{4}, \\
M_{2}(x, y, z) \geq & \frac{[1+G(g y, f y, f y)] G(g z, f x, f x)}{2+G(g x, g y, f y)} \\
= & \frac{[1+G(y, 0,0)] G(7 / 6,1 / 6,1 / 6)}{2+G(7 / 6, y, 0)} \\
= & \frac{2(1+2 y)}{2+y+7 / 6+|y-7 / 6|} \geq \frac{6}{13} .
\end{aligned}
$$


It is clear that

$$
\begin{aligned}
M_{i}(x, y, z) & \geq \frac{6}{13}, \psi\left(\int_{0}^{G(f x, f y, f z)} \varphi(t) d t\right)=3 \int_{0}^{1 / 3} 2 t d t \\
& =\frac{1}{3}<2 \cdot \frac{36}{169}=\eta\left(\int_{0}^{6 / 13} \varphi(t) d t\right) \leq \eta\left(\int_{0}^{M_{i}(x, y, z)} \varphi(t) d t\right) \\
& =\psi\left(\int_{0}^{M_{i}(x, y, z)} \varphi(t) d t\right)-\phi\left(\int_{0}^{M_{i}(x, y, z)} \varphi(t) d t\right), \quad \forall i \in\{1,2\} .
\end{aligned}
$$

Case 7. $z \in[0,1]$ and $x, y \in(1,2]$. It follows that

$$
\begin{aligned}
M_{1}(x, y, z) \geq & \frac{[1+G(g y, g y, f y)] G(g x, g x, f z)}{2[1+G(g y, f x, f y)]} \\
& =\frac{[1+G(7 / 6,7 / 6,1 / 6)] G(7 / 6,7 / 6,0)}{2[1+G(7 / 6,1 / 6,1 / 6)]} \\
& =\frac{(7 / 3)(1+2)}{2(1+2)}=\frac{7}{6}, \\
M_{2}(x, y, z) \geq & \frac{[1+G(g y, f y, f y)] G(g x, f z, f z)}{2[1+G(g y, g z, f y)]} \\
= & \frac{[1+G(7 / 6,1 / 6,1 / 6)] G(7 / 6,0,0)}{2[1+G(7 / 6, z, 1 / 6)]} \\
= & \frac{(7 / 3)(1+2)}{2(1+1+|z-7 / 6|+|z-1 / 6|)} \geq \frac{21}{20} .
\end{aligned}
$$

It is easy to obtain that

$$
\begin{aligned}
& M_{i}(x, y, z) \geq \frac{21}{20} \\
& \psi\left(\int_{0}^{G(f x, f y, f z)} \varphi(t) d t\right)= 3 \int_{0}^{1 / 3} 2 t d t=\frac{1}{3}<2 \cdot \frac{441}{400} \\
&= \eta\left(\int_{0}^{21 / 20} \varphi(t) d t\right) \leq \eta\left(\int_{0}^{M_{i}(x, y, z)} \varphi(t) d t\right) \\
&= \psi\left(\int_{0}^{M_{i}(x, y, z)} \varphi(t) d t\right) \\
&-\phi\left(\int_{0}^{M_{i}(x, y, z)} \varphi(t) d t\right), \quad \forall i \in\{1,2\} .
\end{aligned}
$$

Therefore, (12) and (51) hold. That is, the conditions of Theorems 19 and 20 are fulfilled. It follows from each of Theorems 19 and 20 that $f$ and $g$ have a unique common fixed point in $X$.

However, Theorem 4 cannot be applied to testify the existence of fixed points of the mapping $f$ in $X$. Suppose that there exists $\psi, \phi \in \Phi_{2}$ satisfying the conditions of Theorem
4. In virtue of (4), we infer that

$$
\begin{aligned}
\psi\left(\frac{1}{3}\right)= & \psi\left(G\left(f 1, f 1, f \frac{7}{6}\right)\right) \leq \psi\left(G\left(1,1, \frac{7}{6}\right)\right) \\
& -\phi\left(G\left(1,1, \frac{7}{6}\right)\right)=\psi\left(\frac{1}{3}\right)-\phi\left(\frac{1}{3}\right)<\psi\left(\frac{1}{3}\right),
\end{aligned}
$$

which is a contradiction.

Now we claim that Theorem 5 is useless in proving the existence of common fixed points of the mappings $f$ and $g$ in $X$. Suppose that there exist $\alpha \in[0,1)$ and $\varphi \in \Phi_{1}$ satisfying the conditions of Theorem 5 . Taking advantage of (5), we receive that

$$
\begin{aligned}
\int_{0}^{1 / 3} \varphi(t) d t & =\int_{0}^{G(f 1, f 2, f 2)} \varphi(t) d t \leq \alpha \int_{0}^{G(g 1, g 2, g 2)} \varphi(t) d t \\
& =\alpha \int_{0}^{1 / 3} \varphi(t) d t<\int_{0}^{1 / 3} \varphi(t) d t
\end{aligned}
$$

which is impossible.

\section{Applications}

In this section, we study the existence and uniqueness of common solutions for the below functional equations (72) and nonlinear Volterra integral equations (94) by using the results obtained in Section 3.

Let $U$ and $V$ denote two Banach spaces; $S \subseteq U$ and $D \subseteq V$ signify the state and decision spaces, respectively. $B(S)$ indicates the Banach space of all bounded functions in $S$ with norm

$$
\|h\|=\sup \{|h(x)|: x \in S\}, \quad \forall h \in B(S) .
$$

Define $G:(B(S))^{3} \rightarrow \mathbb{R}^{+}$by

$G(x, y, z)=\max \{\|x-y\|,\|y-z\|,\|z-x\|\}, \quad \forall x, y, z \in B(S)$.

It is clear that $(B(S), G)$ is a complete $G$-metric space.

Consider the functional equations arising in dynamic programming:

$$
\begin{array}{cl}
f(x)=\sup _{y \in D}\{u(x, y)+H(x, y, f(a(x, y)))\}, & \forall x \in S, \\
g(x)=\sup _{y \in D}\{v(x, y)+L(x, y, g(b(x, y)))\}, \quad \forall x \in S,
\end{array}
$$

where $u, v: S \times D \rightarrow \mathbb{R}, a, b: S \times D \rightarrow S$, and $H, L: S \times D \times$ $\mathbb{R} \rightarrow \mathbb{R}$ are mappings. Put

$$
f h(x)=\sup _{y \in D}\{u(x, y)+H(x, y, h(a(x, y)))\}, \quad \forall(x, h) \in S \times B(S) \text {, }
$$


$g h(x)=\sup _{y \in D}\{v(x, y)+L(x, y, h(b(x, y)))\}, \quad \forall(x, h) \in S \times B(S)$.

Theorem 23. Let $u, v: S \times D \rightarrow \mathbb{R}, a, b: S \times D \rightarrow S$, and $H$, $L: S \times D \times \mathbb{R} \rightarrow \mathbb{R}$ satisfy that

(C1) $u, v, H$, and L are bounded;

(C2) $f g h=g$ fh for each $h \in B(S)$ with $f h=g h$;

(C3) $f(B(S)) \subseteq g(B(S))$ and $g(B(S))$ is complete; $B(S)$

(C4) for each $i \in\{1,2,3\}, x \in S, y \in D$, and $h_{1}, h_{2}, h_{3} \in$

$\psi\left(\int_{0}^{\left|H\left(x, y, h_{i}(a(x, y))\right)-H\left(x, y, h_{i t+}(a(x, y))\right)\right|} \varphi(t) d t\right) \leq \psi\left(\int_{0}^{M_{i}^{*}} \varphi(t) d t\right)-\phi\left(\int_{0}^{M_{i}^{*}} \varphi(t) d t\right)$,

where $(\varphi, \psi, \phi) \in \Phi_{1} \times \Phi_{5} \times \Phi_{3}, h_{4}=h_{1}$ and

$$
\begin{aligned}
M_{1}^{*}\left(h_{1}, h_{2}, h_{3}\right)= & \max \left\{G\left(g h_{1}, g h_{2}, g h_{3}\right),\right. \\
& \frac{\left[1+G\left(g h_{1}, g h_{1}, f h_{1}\right)\right] G\left(g h_{2}, g h_{2}, f h_{3}\right)}{2+G\left(g h_{1}, f h_{1}, f h_{2}\right)}, \\
& \frac{\left[1+G\left(g h_{1}, g h_{1}, f h_{1}\right)\right] G\left(g h_{3}, g h_{3}, f h_{2}\right)}{2+G\left(g h_{1}, f h_{1}, f h_{3}\right)}, \\
& \frac{\left[1+G\left(g h_{2}, g h_{2}, f h_{2}\right)\right] G\left(g h_{3}, g h_{3}, f h_{1}\right)}{2+G\left(g h_{2}, f h_{2}, f h_{3}\right)}, \\
& \frac{\left[1+G\left(g h_{2}, g h_{2}, f h_{2}\right)\right] G\left(g h_{1}, g h_{1}, f h_{3}\right)}{2\left[1+G\left(g h_{2}, f h_{1}, f h_{2}\right)\right]}, \\
& \frac{\left[1+G\left(g h_{3}, g h_{3}, f h_{3}\right)\right] G\left(g h_{2}, g h_{2}, f h_{1}\right)}{2\left[1+G\left(g h_{3}, f h_{2}, f h_{3}\right)\right]}, \\
& \frac{\left[1+G\left(g h_{3}, g h_{3}, f h_{3}\right)\right] G\left(g h_{1}, g h_{1}, f h_{2}\right)}{2\left[1+G\left(g h_{3}, f h_{1}, f h_{3}\right)\right]}, \\
& \frac{\left[1+G\left(g h_{1}, g h_{2}, g h_{3}\right)\right] G\left(f h_{1}, f h_{2}, f h_{3}\right)}{1+G\left(g h_{3}, f h_{2}, f h_{2}\right)+G\left(g h_{1}, g h_{2}, g h_{3}\right)}, \\
& \frac{\left[1+G\left(g h_{1}, g h_{2}, g h_{3}\right)\right] G\left(f h_{1}, f h_{2}, f h_{3}\right)}{1+G\left(g h_{2}, f h_{1}, f h_{1}\right)+G\left(g h_{1}, g h_{2}, g h_{3}\right)}, \\
& \left.\frac{\left[1+G\left(g h_{1}, g h_{2}, g h_{3}\right)\right] G\left(f h_{1}, f h_{2}, f h_{3}\right)}{1+G\left(g h_{1}, f h_{3}, f h_{3}\right)+G\left(g h_{1}, g h_{2}, g h_{3}\right)}\right\} .
\end{aligned}
$$

Then, the functional equations (72) have a unique common bounded solution $h^{*} \in B(S)$.

Proof. By virtue of (C1) and (73), we obtain that $f h$ and $g h$ are bounded for each $h \in B(S)$, which yields that $f$ and $g$ are self mappings in $B(S)$. It follows from $\varphi \in \Phi_{1}$ that for each $\varepsilon>0$ there exists $\delta>0$ such that

$$
\int_{C} \varphi(t) d t<\varepsilon, \quad \forall C \subset \mathbb{R}^{+} \text {with } m(C) \leq \delta,
$$

where $m$ denotes the Lebesgue measure. Put $x \in S$ and $h_{1}, h_{2}$ , $h_{3} \in B(S)$. (73) means that there exist $y_{1}, y_{2} \in D$ such that

$$
f h_{1}(x)<u\left(x, y_{1}\right)+H\left(x, y_{1}, h_{1}\left(a\left(x, y_{1}\right)\right)\right)+\delta,
$$

$$
\begin{gathered}
f h_{2}(x)<u\left(x, y_{2}\right)+H\left(x, y_{2}, h_{2}\left(a\left(x, y_{2}\right)\right)\right)+\delta, \\
f h_{1}(x) \geq u\left(x, y_{2}\right)+H\left(x, y_{2}, h_{1}\left(a\left(x, y_{2}\right)\right)\right), \\
f h_{2}(x) \geq u\left(x, y_{1}\right)+H\left(x, y_{1}, h_{2}\left(a\left(x, y_{1}\right)\right)\right) .
\end{gathered}
$$

In terms of (78) and (81), we gain that

$$
\begin{aligned}
& f h_{1}(x)-f h_{2}(x)<H\left(x, y_{1}, h_{1}\left(a\left(x, y_{1}\right)\right)\right) \\
& \quad-H\left(x, y_{1}, h_{2}\left(a\left(x, y_{1}\right)\right)\right)+\delta \leq \mid H\left(x, y_{1}, h_{1}\left(a\left(x, y_{1}\right)\right)\right) \\
& \quad-H\left(x, y_{1}, h_{2}\left(a\left(x, y_{1}\right)\right)\right) \mid+\delta .
\end{aligned}
$$

On account of (79) and (80), we derive that

$$
\begin{aligned}
& f h_{2}(x)-f h_{1}(x)<H\left(x, y_{2}, h_{2}\left(a\left(x, y_{2}\right)\right)\right) \\
& \quad-H\left(x, y_{2}, h_{1}\left(a\left(x, y_{2}\right)\right)\right)+\delta \leq \mid H\left(x, y_{2}, h_{2}\left(a\left(x, y_{2}\right)\right)\right) \\
& \quad-H\left(x, y_{2}, h_{1}\left(a\left(x, y_{2}\right)\right)\right) \mid+\delta .
\end{aligned}
$$

In light of (82) and (83), we get that

$$
\left|f h_{1}(x)-f h_{2}(\mathrm{x})\right|<\max \left\{T_{1}, T_{2}\right\}+\delta,
$$

where

$$
\begin{aligned}
& T_{1}=\left|H\left(x, y_{1}, h_{1}\left(a\left(x, y_{1}\right)\right)\right)-H\left(x, y_{1}, h_{2}\left(a\left(x, y_{1}\right)\right)\right)\right|, \\
& T_{2}=\left|H\left(x, y_{2}, h_{2}\left(a\left(x, y_{2}\right)\right)\right)-H\left(x, y_{2}, h_{1}\left(a\left(x, y_{2}\right)\right)\right)\right| .
\end{aligned}
$$
that

It follows from (75), (84), and $(\varphi, \psi, \phi) \in \Phi_{1} \times \Phi_{5} \times \Phi_{3}$

$$
\begin{aligned}
& \psi\left(\int_{0}^{\left|f h_{1}(x)-f h_{2}(x)\right|} \varphi(t) d t\right) \leq \psi\left(\int_{0}^{\max \left\{T_{1}, T_{2}\right\}+\delta} \varphi(t) d t\right) \\
& =\max \left\{\psi\left(\int_{0}^{T_{1}+\delta} \varphi(t) d t\right), \psi\left(\int_{0}^{T_{2}+\delta} \varphi(t) d t\right)\right\} \\
& =\max \left\{\psi\left(\int_{0}^{T_{1}} \varphi(t) d t+\int_{T_{1}}^{T_{1}+\delta} \varphi(t) d t\right), \psi\right. \\
& \left.\cdot\left(\int_{0}^{T_{2}} \varphi(t) d t+\int_{T_{2}}^{T_{2}+\delta} \varphi(t) d t\right)\right\} \\
& \leq \max \left\{\psi\left(\int_{0}^{T_{1}} \varphi(t) d t\right)+\psi\left(\int_{T_{1}}^{T_{1}+\delta} \varphi(t) d t\right), \psi\right. \\
& \left.\cdot\left(\int_{0}^{T_{2}} \varphi(t) d t\right)+\psi\left(\int_{T_{2}}^{T_{2}+\delta} \varphi(t) d t\right)\right\} \\
& \leq \max \left\{\psi\left(\int_{0}^{T_{1}} \varphi(t) d t\right), \psi\left(\int_{0}^{T_{2}} \varphi(t) d t\right)\right\} \\
& +\max \left\{\psi\left(\int_{T_{1}}^{T_{1}+\delta} \varphi(t) d t\right), \psi\left(\int_{T_{2}}^{T_{2}+\delta} \varphi(t) d t\right)\right\} \\
& \leq \psi\left(\int_{0}^{M_{1}^{*}} \varphi(t) d t\right)-\phi\left(\int_{0}^{M_{1}^{*}} \varphi(t) d t\right)+\psi(\varepsilon) .
\end{aligned}
$$

Taking $\varepsilon \rightarrow 0^{+}$in the above inequalities and using $\psi \in \Phi_{5}$ 
and (70), we infer that

$$
\begin{gathered}
\psi\left(\int_{0}^{\left|f h_{1}(x)-f h_{2}(x)\right|} \varphi(t) d t\right) \leq \psi\left(\int_{0}^{M_{1}^{*}} \varphi(t) d t\right)-\phi\left(\int_{0}^{M_{1}^{*}} \varphi(t) d t\right) \\
\psi\left(\int_{0}^{\left\|f h_{1}-f h_{2}\right\|} \varphi(t) d t\right) \leq \psi\left(\int_{0}^{M_{1}^{*}} \varphi(t) d t\right)-\phi\left(\int_{0}^{M_{1}^{*}} \varphi(t) d t\right) .
\end{gathered}
$$

Similarly, we deduce that

$$
\begin{aligned}
& \psi\left(\int_{0}^{\left\|f h_{2}-f h_{3}\right\|} \varphi(t) d t\right) \leq \psi\left(\int_{0}^{M_{1}^{*}} \varphi(t) d t\right)-\phi\left(\int_{0}^{M_{1}^{*}} \varphi(t) d t\right), \\
& \psi\left(\int_{0}^{\left\|f h_{3}-f h_{1}\right\|} \varphi(t) d t\right) \leq \psi\left(\int_{0}^{M_{1}^{*}} \varphi(t) d t\right)-\phi\left(\int_{0}^{M_{1}^{*}} \varphi(t) d t\right) .
\end{aligned}
$$

It follows that

$$
\begin{aligned}
\psi\left(\int_{0}^{G\left(f h_{1}, f h_{2}, f h_{3}\right)} \varphi(t) d t\right)= & \psi\left(\int_{0}^{\max \left\{\left\|f h_{1}-f h_{2}\right\|,\left\|f h_{2}-f h_{3}\right\|\left\|f h_{3}-f h_{1}\right\|\right\}} \varphi(t) d t\right) \\
= & \max \left\{\psi\left(\int_{0}^{\left\|f h_{1}-f h_{2}\right\|} \varphi(t) d t\right), \psi\right. \\
& \left.\cdot\left(\int_{0}^{\left\|f h_{2}-f h_{3}\right\|} \varphi(t) d t\right), \psi\left(\int_{0}^{\left\|f h_{3}-f h_{1}\right\|} \varphi(t) d t\right)\right\} \\
\leq & \psi\left(\int_{0}^{M_{1}^{*}} \varphi(t) d t\right) \\
& -\phi\left(\int_{0}^{M_{1}^{*}} \varphi(t) d t\right), \quad \forall h_{1}, h_{2}, h_{3} \in B(S) .
\end{aligned}
$$

Consequently, the conditions of Theorem 19 are satisfied. It follows from Theorem 19 that $f$ and $g$ have a unique common fixed point $h^{*} \in B(S)$, that is, the functional equations (72) have a unique common bounded solution $h^{*} \in B(S)$. This completes the proof.

As in the proof of Theorem 23, we obtain similarly the following result and omit its proof.

Theorem 24. Let $u, v: S \times D \rightarrow \mathbb{R}, a, b: S \times D \rightarrow S$, and $H$, $L: S \times D \times \mathbb{R} \rightarrow \mathbb{R}$ satisfy (C1)-(C3) and (C5) for each $i \in\{1$ , 2, 3\}, $x \in S, y \in D$ and $h_{1}, h_{2}, h_{3} \in B(S)$

$$
\psi\left(\int_{0}^{\left|H\left(x, y, h_{i}(a(x, y))\right)-H\left(x, y, h_{i+1}(a(x, y))\right)\right|} \varphi(t) d t\right) \leq \psi\left(\int_{0}^{M_{2}^{*}} \varphi(t) d t\right)-\phi\left(\int_{0}^{M_{2}^{*}} \varphi(t) d t\right),
$$

where $(\varphi, \psi, \phi) \in \Phi_{1} \times \Phi_{5} \times \Phi_{3}, h_{4}=h_{1}$ and

$$
\begin{aligned}
& M_{2}^{*}\left(h_{1}, h_{2}, h_{3}\right)=\max \left\{G\left(g h_{1}, g h_{2}, g h_{3}\right)\right. \text {, } \\
& \frac{\left[1+G\left(g h_{1}, f h_{1}, f h_{1}\right)\right] G\left(g h_{3}, f h_{2}, f h_{2}\right)}{2+G\left(g h_{1}, g h_{2}, f h_{1}\right)}, \\
& \frac{\left[1+G\left(g h_{2}, f h_{2}, f h_{2}\right)\right] G\left(g h_{3}, f h_{1}, f h_{1}\right)}{2+G\left(g h_{1}, g h_{2}, f h_{2}\right)}, \\
& \frac{\left[1+G\left(g h_{3}, f h_{3}, f h_{3}\right)\right] G\left(g h_{1}, f h_{2}, f h_{2}\right)}{2+G\left(g h_{2}, g h_{3}, f h_{3}\right)}, \\
& \frac{\left[1+G\left(g h_{1}, f h_{1}, f h_{1}\right)\right] G\left(g h_{2}, f h_{3}, f h_{3}\right)}{2\left[1+G\left(g h_{1}, g h_{3}, f h_{1}\right)\right]}, \\
& \frac{\left[1+G\left(g h_{2}, f h_{2}, f h_{2}\right)\right] G\left(g h_{1}, f h_{3}, f h_{3}\right)}{2\left[1+G\left(g h_{2}, g h_{3}, f h_{2}\right)\right]}, \\
& \frac{\left[1+G\left(g h_{3}, f h_{3}, f h_{3}\right)\right] G\left(g h_{2}, f h_{1}, f h_{1}\right)}{2\left[1+G\left(g h_{1}, g h_{3}, f h_{3}\right)\right]}, \\
& \frac{\left[1+G\left(g h_{1}, g h_{2}, g h_{3}\right)\right] G\left(f h_{1}, f h_{2}, f h_{3}\right)}{1+G\left(g h_{3}, g h_{3}, f h_{2}\right)+G\left(g h_{1}, g h_{2}, g h_{3}\right)}, \\
& \frac{\left[1+G\left(g h_{1}, g h_{2}, g h_{3}\right)\right] G\left(f h_{1}, f h_{2}, f h_{3}\right)}{1+G\left(g h_{2}, g h_{2}, f h_{1}\right)+G\left(g h_{1}, g h_{2}, g h_{3}\right)}, \\
& \left.\frac{\left[1+G\left(g h_{1}, g h_{2}, g h_{3}\right)\right] G\left(f h_{1}, f h_{2}, f h_{3}\right)}{1+G\left(g h_{1}, g h_{1}, f h_{3}\right)+G\left(g h_{1}, g h_{2}, g h_{3}\right)}\right\} \text {. }
\end{aligned}
$$

Then, the functional equations (72) have a unique common bounded solution $h^{*} \in B(S)$.

Let $C([0, T], \mathbb{R})$ denote the Banach space of all continuous functions in $[0, T]$ with norm

$\|x\|=\sup \{|x(t)|: t \in[0, T]\}, \quad \forall x \in C([0, T], R)$.

Put $X=C([0, T], \mathbb{R})$ and define $G: X \times X \times X \rightarrow \mathbb{R}^{+}$by

$$
G(x, y, z)=\|x-y\|+\|y-z\|+\|z-x\|, \quad \forall x, y, z \in X .
$$

It is obvious that $(X, G)$ is a complete $G$-metric space. Consider the nonlinear Volterra integral equations:

$$
\begin{aligned}
x(t) & =p_{1}(t)+\int_{0}^{t} K_{1}(t, s, x(s)) d s, \forall t \in[0, T], y(t) \\
& =p_{2}(t)+\int_{0}^{t} K_{2}(t, s, y(s)) d s, \forall t \in[0, T],
\end{aligned}
$$

where $T>0$ is a constant, $p_{1}, p_{2}:[0, T] \rightarrow \mathbb{R}$ and $K_{1}, K_{2}$ $:[0, T]^{2} \times \mathbb{R} \rightarrow \mathbb{R}$ are given functions.

Put

$$
f x(t)=p_{1}(t)+\int_{0}^{t} K_{1}(t, s, x(s)) d s, \quad \forall(t, x) \in[0, T] \times X,
$$




$$
g x(t)=p_{2}(t)+\int_{0}^{t} K_{2}(t, s, x(s)) d s, \quad \forall(t, x) \in[0, T] \times X .
$$

Theorem 25. Let $K_{1}, K_{2}:[0, T]^{2} \times \mathbb{R} \rightarrow \mathbb{R}$ and $p_{1}, p_{2}:[0, T]$ $\rightarrow R$ satisfy that

(d1) $K_{1}, K_{2}, p_{1}$, and $p_{2}$ are continuous;

(d2) $f g x=g f x$ for each $x \in X$ with $f x=g x$;

(d3) $f(X) \subseteq g(X)$ and $g(X)$ is complete;

(d4) there exists a continuous function $Q:[0, T] \times[0, T]$ $\rightarrow \mathbb{R}^{+}$such that

$\left|K_{1}(t, s, x(s))-K_{l}(t, s, y(s))\right| \leq Q(t, s)|g x(s)-g y(s)|, \quad \forall t, s \in[0, T], x, y \in X$.

(d5) $\sup _{t \in[0, T]} \int_{0}^{t} Q(t, s) d s \leq 1 / 2$.

Then, the nonlinear Volterra integral equations (94) have a unique common continuous solution in $X$.

Proof. Define $\varphi, \psi, \phi: \mathbb{R}^{+} \rightarrow \mathbb{R}^{+}$by

$$
\varphi(t)=1, \psi(t)=2 t, \phi(t)=t, \quad \forall t \in R^{+} .
$$

Clearly, $(\varphi, \psi, \phi) \in \Phi_{1} \times \Phi_{2} \times \Phi_{3}$. On account of $(d 1)$ and (95), we deduce that for each $x \in X, f x$ and $g x$ are continuous functions in $[0, T]$, that is, $f$ and $g$ are mappings from $X$ into itself. By means of (93), (95), (97), and (d5), we obtain that

$$
\begin{aligned}
\psi\left(\int_{0}^{G(f x, f y, f z)} \varphi(t) d t\right)= & 2 \int_{0}^{G(f x, f y, f z)} \varphi(t) d t=2 G(f x, f y, f z) \\
= & 2(\|f x-f y\|+\|f y-f z\|+\|f z-f x\|) \\
\leq & 2\left(\sup _{t \in[0, T]} \int_{0}^{t}\left|K_{1}(t, s, x(s))-K_{1}(\mathrm{t}, s, y(s))\right| d s\right. \\
& +\sup _{t \in[0, T]} \int_{0}^{t}\left|K_{1}(t, s, y(s))-K_{1}(t, s, z(s))\right| d s \\
& \left.+\sup _{t \in[0, T]} \int_{0}^{t}\left|K_{1}(t, s, z(s))-K_{1}(t, s, x(s))\right| d s\right) \\
\leq & 2\left(\sup _{t \in[0, T]} \int_{0}^{t} Q(t, s)|g x(s)-g y(s)| d s\right. \\
& +\sup _{t \in[0, T]} \int_{0}^{t} Q(t, s)|g y(s)-g z(s)| d s \\
& \left.+\sup _{t \in[0, T]} \int_{0}^{t} Q(t, s)|g z(s)-g x(s)| d s\right) \\
\leq & 2\left(\sup _{t \in[0, T]} \int_{0}^{t} Q(t, s) d s\right)(\|g x-g y\| \\
& +\|g y-g z\|+\|g z-g x\|) \leq G(g x, g y, g z) \\
\leq & M_{i}(x, y, z)=\psi\left(\int_{0}^{M_{i}(x, y, z)} \varphi(t) d t\right)-\phi \\
& +\left(\int_{0}^{M_{i}(x, y, z)} \varphi(t) d t\right), \forall x, y, z \in X, i \in\{1,2\},
\end{aligned}
$$

where $M_{1}(x, y, z)$ and $M_{2}(x, y, z)$ are defined by (13) and (52), respectively. That is, the conditions of Theorems 19 and 20 are fulfilled. Therefore, each of Theorems 19 and 20 guarantees that $f$ and $g$ have a unique common fixed point $x \in X$, which is a unique common continuous solution of the nonlinear Volterra integral equations (94) in $X$. This completes the proof.

\section{Data Availability}

No data were used to support this study.

\section{Conflicts of Interest}

The authors declare that they have no conflicts of interest regarding the publication of this paper.

\section{Acknowledgments}

This work was financially supported by the Science and Research Project Foundation of Liaoning Province Education Department (grant numbers LQN201902 and LJC202003).

\section{References}

[1] H. Aydi, "A fixed point result involving a generalized weakly contractive condition in G-metric spaces," Bulletin of Mathematical Analysis and Applications, vol. 3, pp. 180-188, 2011.

[2] H. Aydi, "A common fixed point of integral type contraction in generalized metric spaces," Journal of Advanced Mathematical Studies, vol. 5, pp. 111-117, 2012.

[3] M. Asadi and P. Salimi, "Some fixed point and common fixed point theorems on G-metric spaces," Nonlinear Functional Analysis and Applications, vol. 21, pp. 523-530, 2016.

[4] A. Branciari, "A fixed point theorem for mappings satisfying a general contractive condition of integral type," International Journal of Mathematics and Mathematical Sciences, vol. 29, pp. 531-536, 2002.

[5] A. Erduran and I. Altun, "Fixed point theorems for weakly contractive mappings on G-metric spaces and a homotopy result," International Journal of Nonlinear Analysis and Applications, vol. 3, pp. 1-8, 2012.

[6] S. Kumar, R. Chugh, and R. Kumar, "Fixed point theorem for compatible mappings satisfying a contractive condition of integral type," Soochow Journal of Mathematics, vol. 33, pp. 181-185, 2007.

[7] Z. Liu, X. Li, S. M. Kang, and S. Y. Cho, "Fixed point theorems for mappings satisfying contractive conditions of integral type and applications," Fixed point theory and Applications, vol. 2011, 18 pages, 2011.

[8] Z. Liu, J. Li, and S. M. Kang, "Fixed point theorems of contractive mappings of integral type," Fixed point theory and Applications, vol. 2013, 17 pages, 2013.

[9] Z. Mustafa and B. Sims, "A new approach to generalized metric spaces," Journal of Nonlinear and convex Analysis, vol. 7, pp. 289-297, 2006.

[10] S. K. Mohanta, "Common fixed points for generalized weakly contractive mappings in G-metric spaces," International Journal of Mathematics Trends and Technology, vol. 5, pp. 88-96, 2014 . 
[11] T. Phaneendra and K. K. Swamy, "Unique fixed point in Gmetric space through greatest lower bound properties," Novi Sad Journal of Mathematics, vol. 43, pp. 107-115, 2013.

[12] B. E. Rhoades, "Some theorems on weakly contractive maps," Nonlinear Analysis, vol. 47, pp. 2683-2693, 2001.

[13] R. A. Rashwan and S. M. Saleh, "A common fixed point theorem of three $(\psi, \varphi)$-weakly contractive mapping in G-metric spaces," Facta Universitatis, Series: Mathematics and Informatics, vol. 28, pp. 323-334, 2013.

[14] W. Sintunavarat and P. Kumam, "Generalized common fixed point theorems in complex valued metric spaces and applications," Journal of Inequalities and Applications, vol. 2012, Article ID 84, 12 pages, 2012.

[15] M. Shoaib, M. Sarwar, K. Shah, and P. Kumam, "Fixed point results and its applications to the systems of non-linear integral and differential equations of arbitrary order," Journal of Nonlinear Sciences and Applications, vol. 9, pp. 4949-4962, 2016.

[16] R. P. Agarwal, E. Karapinar, D. O'Regan, and A. F. RoldanLopez-de-Hierro, Fixed Point Theory in Metric Type Spaces, Springer, 2015.

[17] S. Chandok, Z. Mustafa, and M. Postolache, "Coupled common fixed point results for mixed g-monotone mapps in partially ordered G-metric spaces," University Politehnica of Bucharest Scientific Bulletin A, vol. 75, no. 4, pp. 13-26, 2013.

[18] S. Chandok and S. Manro, "Existence of fixed points in quasi metric spaces," Communications, vol. 69, pp. 266-275, 2019.

[19] V. Gupta, R. K. Saini, and R. Deep, "Some fixed point results in G-metric space involving generalised altering distances," International Journal of Applied Nonlinear Science, vol. 3, no. 1, pp. 66-76, 2018.

[20] V. Gupta and R. Deep, "Some fixed point theorems in Gmetric and fuzzy metric spaces using E.A property," Journal of Information and Computing Science, vol. 10, no. 2, pp. 8389, 2015.

[21] V. Gupta and N. Mani, "Existence and uniqueness of fixed point for contractive mapping of integral type," International Journal of Computing Science and Mathematics, vol. 4, no. 1, pp. 72-83, 2013.

[22] M. Jleli and B. Samet, "Remarks on G-metric spaces and fixed point theorems," Fixed Point Theory and Applications, vol. 2012, 7 pages, 2012. 